\title{
Legendre wavelets with scaling in time-delay systems
}

\author{
Iman Malmir* \\ Department of Aerospace Engineering, MUT University of Technology, Tehran, Iran
}

\begin{abstract}
This research presents the integration, product, delay and inverse time operational matrices of Legendre wavelets with an arbitrary scaling parameter and illustrates how to design this parameter in order to improve their accuracy and capability in handling optimal control and analysis of time-delay systems. Using the presented Legendre wavelets, the piecewise delay operational matrix is derived to develop the applicability of Legendre wavelets in systems with piecewise constant time-delays or time-varying delays. With the aid of these matrices, the new Legendre wavelets method is applied on linear time-delay systems. The reliability and efficiency of the method are demonstrated by some numerical experiments.
\end{abstract}

Keywords Legendre wavelets with an arbitrary scale; Legendre wavelet method; piecewise constant delay systems; timedelay systems; Legendre wavelets operational matrix

AMS 2010 subject classifications 41A10; $65 \mathrm{~T} 60$

DOI: $10.19139 /$ soic.v7i1.460

\section{Introduction}

Wavelets as mathematical functions [1], when applied to time-delay systems, have advantages over orthogonal functions. One of the most useful wavelets as Legendre wavelets are constructed from Legendre polynomials which play an important role in engineering modeling [2,3]. In [4], we have mentioned drawbacks of the conventional Chebyshev wavelets (CCWs) in the analysis and optimal control of time-delay systems. Also we have shown the advantages of Chebyshev wavelets with scaling over orthogonal functions [5] in problems arising in such systems. By doing a similar experiment as in [6,7], we can observe similar drawbacks in the use of the conventional Legendre wavelets (CLWs). The main issue of CLW method is that to model the delayed terms of many time-delay systems, we have to approximate the values of delays, for example by using greatest integer values (see [8]), which this issue causes errors in the obtained results. The conventional definition of Legendre wavelets which has been given in $[8,11-27]$ as

$$
\phi_{n m}(t)= \begin{cases}2^{\frac{k}{2}} \sqrt{m+\frac{1}{2}} P_{m}\left(2^{k} t-2 n+1\right), & \frac{n-1}{2^{k-1}} \leq t \leq \frac{n}{2^{k-1}} \\ 0, & \text { otherwise, }\end{cases}
$$

where $P_{m}$ are the well-known Legendre polynomials, $n=1,2, \ldots, 2^{k-1}$ and $m$ is the order of $P_{m}$, does not always present acceptable accuracy when applied to time-delay systems because we often find $2^{k-1} h \notin \mathbb{N}$, where $h$ is the time-delay. In order to deal with this problem, we have two ways:

1. We can apply the method described in [6], using large $k$, which provides an acceptable result. This application, however, has many disadvantages.

2. We can use greatest integer value of delay as $\left[2^{k-1} h\right]+1$ which, as we shall see later, does not provide accurate results.

\footnotetext{
*Correspondence to: Iman Malmir (iman.malmir@ outlook.com).
}

ISSN 2310-5070 (online) ISSN 2311-004X (print)

Copyright (C) 2019 International Academic Press 
Generally, the following disadvantages of CLWs motivate us to use another definition of these wavelets:

- It may not always be possible to solve a variety of problems related to delay models with low values of $k$.

- The method has a little sensitivity to small changes in time delays, that is, by making small changes in time delays, we have to use large values of $k$ to see its effect on the behavior of systems.

- Their definition cannot be used to get other operational matrices like the piecewise delay operational matrix.

These are some practical limitations of CLW method in time-delay systems. In this work, to eliminate the source of error and to increase the applicability of Legendre wavelets, we use a flexible definition of Legendre wavelets and then introduce the useful matrices which are required for the analysis and optimal control of general linear systems with delays and piecewise constant time delays. To show the advantages and accuracy of the proposed method, we compare the results obtained by this method with those found by CLW method and other methods.

We present the concepts of the new Legendre wavelets in Section 2 and since the previous operational matrices have been defined for CLWs, we continue our discussion for deriving their operational matrices in general forms. Then in Section 3, we use the findings to the optimal control and analysis of general linear systems with different kinds of delays such as multiple delays and piecewise constant delay. Numerical examples are solved in Section 4 to demonstrate the effectiveness of the proposed method and one can see the fact that significant improvements in accuracy and capability of Legendre wavelets are obtained.

\section{Preliminaries}

\subsection{Legendre wavelets with arbitrary scaling parameters}

Legendre polynomials of the first kind $P_{m}(x)$ are polynomials in the independent variable $x$ of order $m$ defined as the solutions of the DE

$$
\left(1-x^{2}\right) P_{m}^{\prime \prime}(x)-2 x P_{m}^{\prime}(x)+m(m+1) P_{m}(x)=0 .
$$

Some of the important relations of Legendre polynomials [3] are

$$
\begin{gathered}
\int_{-1}^{1} P_{m}(x) P_{m^{\prime}}(x) d x=\left\{\begin{array}{cl}
\frac{2}{2 m+1}, & m^{\prime}=m \\
0, & m^{\prime} \neq m,
\end{array}\right. \\
\left(x^{2}-1\right) P_{m}^{\prime}(x)=m x P_{m}(x)-m P_{m-1}(x), \\
(m+1) P_{m+1}(x)-(2 m+1) x P_{m}(x)+m P_{m-1}(x)=0, \\
P_{m}(-x)=(-1)^{m} P_{m}(x) ;
\end{gathered}
$$

the recurrence relation (4) is known as Bonnet's recursion formula [2]. The Legendre polynomials $P_{m}(x)$ are orthogonal with respect to the weight function $w(x)=1$. We can expand a continuous function $f(x)$ on $[-1,1]$ in a Legendre series as

$$
f(x)=\sum_{m=0}^{\infty} a_{m} P_{m}(x) \text {, where } a_{m}=\frac{2 m+1}{2} \int_{-1}^{1} f(x) P_{m}(x) d x .
$$

\section{Definition 1}

\section{The arbitrary scaled Legendre wavelets (ASLWs)}

Dilations and translations of the Mother function $\phi(t)$ define an orthogonal basis, the wavelets $\phi_{s d}(t)$, where $s \neq 0$ and $d$ are integers that scale and dilate the mother function $\phi(t)$ to generate the wavelets [1]

$$
\phi_{s d}(t)=|s|^{-1 / 2} \phi\left(\frac{t-d}{s}\right) .
$$


By selecting

$$
d=(2 n-1) / 2 \xi^{k-1}, s=1 / 2 \xi^{k-1}
$$

and $\sqrt{\frac{2 m+1}{2}}$ from (2) for orthonormality, the arbitrary scaled Legendre wavelets (ASLWs) $\phi_{n m}^{\xi}=\phi(\xi, k, n, m, t)$ with five arguments are defined as

$$
\phi_{n m}^{\xi}(t)= \begin{cases}\sqrt{\xi^{k-1}} c_{m} P_{m}\left(2 \xi^{k-1} t-2 n+1\right), & t \in\left[\frac{n-1}{\xi^{k-1}}, \frac{n}{\xi^{k-1}}\right] \\ 0, & t \notin\left[\frac{n-1}{\xi^{k-1}}, \frac{n}{\xi^{k-1}}\right],\end{cases}
$$

where

$\xi \in \mathbb{N}_{\geq 2}$ is an arbitrarily selected scaling parameter,

$k \in \mathbb{N}_{\geq 2}$ together with $\xi$ specify the number of subintervals,

$n=1,2, \ldots, \xi^{k-1}$ refers to the number of subinterval and specifies the location of the subinterval,

$m=0,1, \ldots, M-1$ is the degree of $P_{m}$ and $c_{m}$ is

$$
c_{m}=\sqrt{2 m+1}
$$

$t \in[0,1]$ is as an independent variable.

These Legendre wavelets are an orthonormal set with respect to the weight functions $w(t)=1$. As we shall see later, by selecting an appropriate value of the scaling parameter, ASLW method may provide the exact solutions of delay differential equations and accurate solutions of time-delay optimization problems. Ref. [10] by defining a sequence of spaces has constructed an orthonormal base to solve the Laplace equations which is similar with this base, but we can see changes in $\mu$ does not have noticeable effects on errors of solutions.

\section{Definition 2}

\section{ASLWs expansion}

The Legendre wavelets expansion of a function $f$ defined on the interval $[0,1]$ is given by

$$
f(t)=\sum_{n=1}^{N} \sum_{m=0}^{\infty} f_{n m}^{\xi} \phi_{n m}^{\xi}(t)
$$

where $N$ is large enough. If we truncate (8) with, say, the $(M-1)$ th term in $\xi^{k-1}$ subintervals, then

$$
f(t) \cong f_{\xi k M}(t)=\sum_{n=1}^{\xi^{k-1}} \sum_{m=0}^{M-1} f_{n m}^{\xi} \phi_{n m}^{\xi}(t)=\mathbf{f}_{\xi} \boldsymbol{\Phi}_{\xi}(t),
$$

where $\mathbf{f}_{\xi}$ and $\boldsymbol{\Phi}_{\xi}(t)$ are $1 \times \xi^{k-1} M$ and $\xi^{k-1} M \times 1$ vectors and

$$
\begin{gathered}
\mathbf{f}_{\xi}=\left[f_{10}^{\xi}, \ldots, f_{1 M-1}^{\xi}, f_{20}^{\xi}, \ldots, f_{2 M-1}^{\xi}, \ldots, f_{\xi^{k-1} 0}^{\xi}, \ldots, f_{\xi^{k-1} M-1}^{\xi}\right], \\
\boldsymbol{\Phi}_{\xi}(t)=\left[\phi_{10}^{\xi}(t), \ldots, \phi_{1 M-1}^{\xi}(t), \phi_{20}^{\xi}(t), \ldots, \phi_{2 M-1}^{\xi}(t), \ldots, \phi_{\xi^{k-1} 0}^{\xi}(t), \ldots, \phi_{\xi^{k-1} M-1}^{\xi}(t)\right]^{\top} .
\end{gathered}
$$

$\left\{f_{n m}^{\xi}\right\}$ are constant coefficients. Finally, we show how to find these coefficients. From (6) we see that when $t \in$ $\left[\frac{n-1}{\xi^{k-1}}, \frac{n}{\xi^{k-1}}\right]$ we have $f(t)=f_{n}(t)=\sum_{m=0}^{M-1} f_{n m}^{\xi} \phi_{n m}^{\xi}(t)$. With multiplying $f_{n}(t)$ by $\phi_{n^{\prime} m^{\prime}}^{\xi}(t)$ and then integrating both sides from $\frac{n-1}{\xi^{k-1}}$ to $\frac{n}{\xi^{k-1}}$, we can write

$$
\int_{\frac{n-1}{\xi^{k-1}}}^{\frac{n}{\xi^{k-1}}} f(t) \phi_{n^{\prime} m^{\prime}}^{\xi}(t) d t=\sum_{m=0}^{M-1} f_{n m}^{\xi} \int_{\frac{n-1}{\xi^{k-1}}}^{\frac{n}{\xi^{k-1}}} \phi_{n m}^{\xi}(t) \phi_{n^{\prime} m^{\prime}}^{\xi}(t) d t,
$$


where $m^{\prime}=0,1, \ldots, M-1$ and $n^{\prime}=1,2, \ldots, \xi^{k-1}$; substituting $x=2 \xi^{k-1} t-2 n+1$ and also knowing the fact that $\phi_{n m}(t) \phi_{n^{\prime} m^{\prime}}(t)=0$ when $n^{\prime} \neq n$, yields

$$
\begin{aligned}
\int_{\frac{n-1}{\xi^{k-1}}}^{\frac{n}{\xi^{k-1}}} f(t) \phi_{n m^{\prime}}^{\xi}(t) d t & =\sum_{m=0}^{M-1} f_{n m}^{\xi} \int_{\frac{n-1}{\xi^{k-1}}}^{\frac{n}{\xi^{k-1}}} \phi_{n m}^{\xi}(t) \phi_{n m^{\prime}}^{\xi}(t) d t \\
& =\frac{1}{2} \sum_{m=0}^{M-1} f_{n m}^{\xi} c_{m} c_{m^{\prime}} \int_{-1}^{1} P_{m}(x) P_{m^{\prime}}(x) d x \\
& =\frac{1}{2} f_{n m}^{\xi} c_{m}^{2}\left\{\begin{array}{cl}
\frac{2}{2 m+1}, & m^{\prime}=m \\
0, & m^{\prime} \neq m .
\end{array}\right.
\end{aligned}
$$

Thus from (7) we can obtain the constant coefficients as follows

$$
f_{n m}^{\xi}=\int_{\frac{n-1}{\xi^{k-1}}}^{\frac{n}{\xi^{k-1}}} f(t) \phi_{n m}^{\xi}(t) d t .
$$

\subsection{The integration operational matrix of Legendre wavelets}

We express the integration of the new Legendre wavelet vector on $[0, t]$ as

$$
\int_{0}^{t} \boldsymbol{\Phi}_{\xi}(\vartheta) d \vartheta \cong \mathbf{P}_{\xi} \boldsymbol{\Phi}_{\xi}(t)
$$

where $\mathbf{P}_{\xi}$ is the $\xi^{k-1} M \times \xi^{k-1} M$ operational matrix of integration of ASLWs. By a procedure similar to that in [4], we can derive this matrix. So, if $\frac{n-1}{\xi^{k-1}} \leq t<\frac{n}{\xi^{k-1}}$, then:

1. for $m=0$

$$
\begin{aligned}
\int_{\frac{n-1}{\xi^{k-1}}}^{t} \phi_{n 0}^{\xi}(\vartheta) d \vartheta & =\sqrt{\xi^{k-1}}\left(t+\frac{-n+1}{\xi^{k-1}}\right)=\sqrt{\xi^{k-1}}\left(\frac{1}{\sqrt{\xi^{k-1}}} \frac{1}{2 \xi^{k-1}} \phi_{n 0}^{\xi}(t)+\frac{1}{\sqrt{3} \sqrt{\xi^{k-1}}} \frac{1}{2 \xi^{k-1}} \phi_{n 1}^{\xi}(t)\right) \\
& =\frac{1}{2 \xi^{k-1}}\left[1, \frac{1}{\sqrt{3}}, 0,0, \ldots, 0\right]\left[\phi_{n 0}^{\xi}(t), \phi_{n 1}^{\xi}(t), \phi_{n 2}^{\xi}(t), \ldots, \phi_{n M-1}^{\xi}(t)\right]^{\top} .
\end{aligned}
$$

2. for $m \geq 1$, we first express (1) in self-adjoint form

$$
\frac{d}{d x}\left(\left(1-x^{2}\right) P_{m}^{\prime}(x)\right)+m(m+1) P_{m}(x)=0 .
$$

Then setting $x=2 \xi^{k-1} \vartheta-2 n+1$ and $x_{t}=2 \xi^{k-1} t-2 n+1$ and using (14), (3) and (4), yields

$$
\begin{aligned}
& \int_{\frac{n-1}{\xi^{k-1}}}^{t} \phi_{n m}^{\xi}(\vartheta) d \vartheta=\sqrt{\xi^{k-1}} c_{m} \int_{\frac{n-1}{\xi^{k-1}}}^{t} P_{m}\left(2 \xi^{k-1} \vartheta-2 n+1\right) d \vartheta=\sqrt{\xi^{k-1}} c_{m} \frac{1}{2 \xi^{k-1}} \int_{-1}^{x_{t}} P_{m}(x) d x \\
&=\left.c_{m} \frac{1}{2 \sqrt{\xi^{k-1}}} \frac{1}{m(m+1)}\left(\left(x^{2}-1\right) P_{m}^{\prime}(x)\right)\right|_{-1} ^{x_{t}} \\
&=c_{m} \frac{1}{2 \sqrt{\xi^{k-1}}} \frac{1}{m(m+1)}\left(x_{t}^{2}-1\right) P_{m}^{\prime}\left(x_{t}\right) \\
&=c_{m} \frac{1}{2 \sqrt{\xi^{k-1}}} \frac{1}{m(m+1)}\left(m x_{t} P_{m}\left(x_{t}\right)-m P_{m-1}\left(x_{t}\right)\right) \\
&=c_{m} \frac{1}{2 \sqrt{\xi^{k-1}}} \frac{1}{m+1}\left(\frac{m+1}{2 m+1} P_{m+1}\left(x_{t}\right)+\frac{m}{2 m+1} P_{m-1}\left(x_{t}\right)-P_{m-1}\left(x_{t}\right)\right) \\
&=c_{m} \frac{1}{2 \sqrt{\xi^{k-1}}}\left(-\frac{1}{2 m+1} P_{m-1}\left(2 \xi^{k-1} t-2 n+1\right)+\frac{1}{2 m+1} P_{m+1}\left(2 \xi^{k-1} t-2 n+1\right)\right) \\
&=\frac{1}{2 \sqrt{\xi^{k-1}}}\left(-\frac{1}{\sqrt{2 m+1}} \frac{1}{\sqrt{2 m-1}} \frac{1}{\sqrt{\xi^{k-1}}} \phi_{n m-1}^{\xi}(t)+\frac{1}{\sqrt{2 m+1}} \frac{1}{\sqrt{2 m+3}} \frac{1}{\sqrt{\xi^{k-1}}} \phi_{n m+1}^{\xi}(t)\right) \\
&=\frac{1}{2 \xi^{k-1}}[\underbrace{0,0, \ldots, 0}_{m-1},-\frac{1}{\sqrt{4 m^{2}-1}}, 0, \frac{1}{\sqrt{4 m^{2}+8 m+3}}, 0,0, \ldots, 0] \\
&
\end{aligned}
$$


If $t=\frac{n}{\xi^{k-1}}$, then:

1. for $m=0$

$$
\begin{aligned}
\int_{\frac{n-1}{\xi^{k-1}}}^{t} \phi_{n 0}^{\xi}(\vartheta) d \vartheta & =\sqrt{\xi^{k-1}}\left(\frac{n}{\xi^{k-1}}-\frac{n-1}{\xi^{k-1}}\right)=\sqrt{\xi^{k-1}} \frac{2}{2 \xi^{k-1}} \sum_{\eta=n+1}^{\xi^{k-1}} \frac{1}{\sqrt{\xi^{k-1}}} \phi_{\eta 0}^{\xi}(t) \\
& =\frac{1}{2 \xi^{k-1}} \sum_{\eta=n+1}^{\xi^{k-1}}[2,0,0, \ldots, 0]\left[\phi_{\eta 0}^{\xi}(t), \phi_{\eta 1}^{\xi}(t), \phi_{\eta 2}^{\xi}(t), \ldots, \phi_{\eta M-1}^{\xi}(t)\right]^{\top} .
\end{aligned}
$$

2. for $m \geq 1$

$$
\begin{aligned}
\int_{\frac{n-1}{\xi^{k-1}}}^{t} \phi_{n m}^{\xi}(\vartheta) d \vartheta & =\sqrt{\xi^{k-1}} c_{m} \int_{\frac{n-1}{\xi^{k-1}}}^{\frac{n}{\xi^{k-1}}} P_{m}\left(2 \xi^{k-1} \vartheta-2 n+1\right) d \vartheta=\sqrt{\xi^{k-1}} c_{m} \frac{1}{2 \xi^{k-1}} \int_{-1}^{1} P_{m}(x) d x \\
& =\left.c_{m} \frac{1}{2 \sqrt{\xi^{k-1}}} \frac{1}{m(m+1)}\left(\left(x^{2}-1\right) P_{m}^{\prime}(x)\right)\right|_{-1} ^{1} \\
& =0 \\
& =\frac{1}{2 \xi^{k-1}} \sum_{\eta=n+1}^{\xi^{k-1}}[0,0,0, \ldots, 0,0]\left[\phi_{\eta 0}^{\xi}(t), \phi_{\eta 1}^{\xi}(t), \phi_{\eta 2}^{\xi}(t), \ldots, \phi_{\eta M-1}^{\xi}(t)\right]^{\top} .
\end{aligned}
$$

So

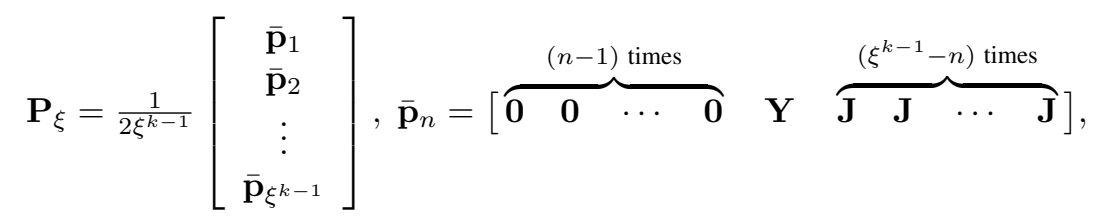

where $\mathbf{Y}$ and $\mathbf{J}$ are matrices of order $M$ and given by

$$
\mathbf{Y}=\left[\begin{array}{ccccccccc}
1 & \frac{1}{\sqrt{3}} & 0 & 0 & 0 & \cdots & 0 & 0 & 0 \\
-\frac{1}{\sqrt{3}} & 0 & \frac{1}{\sqrt{15}} & 0 & 0 & \cdots & 0 & 0 & 0 \\
0 & -\frac{1}{\sqrt{15}} & 0 & \frac{1}{\sqrt{35}} & 0 & \cdots & 0 & 0 & 0 \\
0 & 0 & -\frac{1}{\sqrt{35}} & 0 & \frac{1}{\sqrt{63}} & \cdots & 0 & 0 & 0 \\
\vdots & \vdots & \vdots & \vdots & \vdots & \ddots & \vdots & \vdots & \vdots \\
0 & 0 & 0 & 0 & 0 & \cdots & -\frac{1}{\sqrt{4(M-2)^{2}-1}} \\
0 & 0 & 0 & 0 & 0 & \cdots & 0 & -\frac{1}{\sqrt{4(M-1)^{2}-1}} & 0
\end{array}\right], \mathbf{J}=\left[\begin{array}{ccccc}
2 & 0 & 0 & \cdots & 0 \\
0 & 0 & 0 & \cdots & 0 \\
0 & 0 & 0 & \cdots & 0 \\
0 & 0 & 0 & \cdots & 0 \\
\vdots & \vdots & \vdots & \ddots & \vdots \\
0 & 0 & 0 & \cdots & 0
\end{array}\right] .
$$

\subsection{The integration matrix of the product of Legendre wavelets on [0,1]}

The integration matrix of the product of two new Legendre wavelet function vectors on $[0,1]$ is obtained from $\int_{0}^{1} \boldsymbol{\Phi}_{\xi}(t) \boldsymbol{\Phi}_{\xi}^{\top}(t) d t=\int_{0}^{1}\left[\phi_{10}^{\xi}(t), \ldots, \phi_{1 M-1}^{\xi}(t), \ldots, \phi_{\xi^{k-1} M-1}^{\xi}(t)\right]^{\top}\left[\phi_{10}^{\xi}(t), \ldots, \phi_{1 M-1}^{\xi}(t), \ldots, \phi_{\xi^{k-1} M-1}^{\xi}(t)\right] d t$.

Knowing

$$
\int_{\frac{n-1}{\xi^{k-1}}}^{\frac{n}{\xi^{k-1}}} \phi_{n m}^{\xi}(t) \phi_{n m^{\prime}}^{\xi}(t) d t=\frac{2 m+1}{2} \int_{-1}^{1} P_{m}(x) P_{m^{\prime}}(x) d x= \begin{cases}1, & m^{\prime}=m \\ 0, & m^{\prime} \neq m\end{cases}
$$

and denoting the identity matrix by $\mathbf{I}$, we have

$$
\int_{0}^{1} \boldsymbol{\Phi}_{\xi}(t) \boldsymbol{\Phi}_{\xi}^{\top}(t) d t=\mathbf{I}_{\xi^{k-1} M}
$$




\subsection{The product operational matrix of Legendre wavelets}

Using the relations of Legendre polynomials, we can find

$$
\mathbf{f}_{\xi} \boldsymbol{\Phi}_{\xi}(t) \boldsymbol{\Phi}_{\xi}^{\top}(t) \cong \boldsymbol{\Phi}_{\xi}^{\top}(t) \tilde{\mathbf{f}}_{\xi},
$$

where $\tilde{\mathbf{f}}_{\xi}$ is the $\xi^{k-1} M \times \xi^{k-1} M$ product operational matrix of ASLWs of $\mathbf{f}_{\xi}$. Using a procedure similar to what we did in [7] and Definition 1, we conclude that $\tilde{\mathbf{f}}_{\xi}$ is in the from $\tilde{\mathbf{f}}_{\xi}=z \cdot \operatorname{blkdiag}\left(\tilde{\mathbf{f}}_{1}, \tilde{\mathbf{f}}_{2}, \ldots, \tilde{\mathbf{f}}_{\xi^{k-1}}\right)$. To evaluate $\tilde{\mathbf{f}}_{\xi}$ and $z$, we let $\tilde{\mathbf{f}}_{n}=\left[\tilde{f}_{u v}\right]$, where $u, v=1,2, \ldots, M$. The product of two Legendre polynomials is given by (see [9, 14])

$$
P_{m}(x) P_{m^{\prime}}(x)=\sum_{\alpha=0}^{m^{\prime}} \frac{\Im_{\alpha} \Im_{m-\alpha} \Im_{m^{\prime}-\alpha}}{\Im_{m+m^{\prime}-\alpha}} \frac{2 m+2 m^{\prime}-4 \alpha+1}{2 m+2 m^{\prime}-2 \alpha+1} P_{m+m^{\prime}-2 \alpha}(x),
$$

where $m \geq m^{\prime}, \Im_{\alpha}=\frac{(2 \alpha-1) ! !}{\alpha !}$ and !! is the double factorial. Hence from (18) and (6) we can write

$$
\begin{aligned}
\phi_{n m}^{\xi}(t) \phi_{n m^{\prime}}^{\xi}(t) & =\xi^{k-1} \sqrt{(2 m+1)\left(2 m^{\prime}+1\right)} P_{m}(x) P_{m^{\prime}}(x) \\
& =\xi^{k-1} \sqrt{(2 m+1)\left(2 m^{\prime}+1\right)} \sum_{\alpha=0}^{m^{\prime}} \frac{\Im_{\alpha} \Im_{m-\alpha} \Im_{m^{\prime}-\alpha}}{\Im_{m+m^{\prime}-\alpha}} \frac{2 m+2 m^{\prime}-4 \alpha+1}{2 m+2 m^{\prime}-2 \alpha+1} P_{m+m^{\prime}-2 \alpha}(x) \\
& =\sqrt{\xi^{k-1}} \sqrt{(2 m+1)\left(2 m^{\prime}+1\right)} \sum_{\alpha=0}^{m^{\prime}} \frac{\Im_{\alpha} \Im_{m-\alpha} \Im_{m^{\prime}-\alpha}}{\Im_{m+m^{\prime}-\alpha}} \frac{2 m+2 m^{\prime}-4 \alpha+1}{2 m+2 m^{\prime}-2 \alpha+1} \frac{1}{\sqrt{2\left(m+m^{\prime}-2 \alpha\right)+1}} \phi_{n m+m^{\prime}-2 \alpha}^{\xi}(x) \\
& =\sqrt{\xi^{k-1}} \sum_{\alpha=0}^{m^{\prime}} \wp_{m m^{\prime} \alpha} \phi_{n m+m^{\prime}-2 \alpha}^{\xi}(x),
\end{aligned}
$$

where we set $\wp_{m m^{\prime} \alpha}=\frac{\Im_{\alpha} \Im_{m-\alpha} \Im_{m^{\prime}-\alpha}}{\Im_{m+m^{\prime}-\alpha}} \frac{2 m+2 m^{\prime}-4 \alpha+1}{2 m+2 m^{\prime}-2 \alpha+1} \sqrt{\frac{(2 m+1)\left(2 m^{\prime}+1\right)}{2\left(m+m^{\prime}-2 \alpha\right)+1}}$. If $m+m^{\prime}-2 \alpha \geq M$, then we must take $\wp_{m m^{\prime} \alpha}=0$. By equating coefficients of the same wavelets on both sides of (17), we find, in general,

$$
\tilde{\mathbf{f}}_{\xi}=\sqrt{\xi^{k-1}} \cdot \operatorname{blkdiag}\left(\tilde{\mathbf{f}}_{1}, \tilde{\mathbf{f}}_{2}, \ldots, \tilde{\mathbf{f}}_{\xi^{k-1}}\right)
$$

where

$$
\begin{aligned}
& \tilde{\mathbf{f}}_{n} \cong\left[\begin{array}{ccccc}
f_{n 0}^{\xi} & f_{n 1}^{\xi} & f_{n 2}^{\xi} & \cdots & f_{n M-1}^{\xi} \\
f_{n 1}^{\xi} & f_{n 0}^{\xi}+\wp_{110} f_{n 2}^{\xi} & \wp_{211} f_{n 1}^{\xi}+\wp_{210} f_{n 3}^{\xi} & \cdots & \wp_{M-111} f_{n M-2}^{\xi} \\
f_{n 2}^{\xi} & \wp_{211} f_{n 1}^{\xi}+\wp_{210} f_{n 3}^{\xi} & f_{n 0}^{\xi}+\wp_{221} f_{n 2}^{\xi}+\wp_{220} f_{n 4}^{\xi} & \cdots & \wp_{M-122} f_{n M-3}^{\xi}+\wp_{M-121} f_{n M-1}^{\xi} \\
\vdots & \vdots & \vdots & \ddots & \vdots \\
f_{n M-1}^{\xi} & \wp_{M-111} f_{n M-2}^{\xi} & \wp_{M-1} 22 f_{n M-3}^{\xi}+\wp_{M-121} f_{n M-1}^{\xi} & \cdots & f_{n 0}^{\xi}+\wp_{M-1}{ }_{M-1} M-2 f_{n 2}^{\xi}+\ldots+\wp_{M-1} M-1 \beta f_{n \gamma}^{\xi}
\end{array}\right], \\
& \wp_{m m^{\prime} \alpha}=\frac{\Im_{\alpha} \Im_{m-\alpha} \Im_{m^{\prime}-\alpha}}{\Im_{m+m^{\prime}-\alpha}} \frac{2 m+2 m^{\prime}-4 \alpha+1}{2 m+2 m^{\prime}-2 \alpha+1} \sqrt{\frac{(2 m+1)\left(2 m^{\prime}+1\right)}{2\left(m+m^{\prime}-2 \alpha\right)+1}}, \beta=\left\{\begin{array}{ll}
M / 2, & M \text { even } \\
(M-1) / 2, & M \text { odd }
\end{array}, \gamma= \begin{cases}M-2, & M \text { even } \\
M-1, & M \text { odd. }\end{cases} \right.
\end{aligned}
$$

\subsection{The delay operational matrix of Legendre wavelets}

The delay Legendre scaling function vector $\boldsymbol{\Phi}_{\xi}\left(t-h_{\iota}\right)$ can be expressed in the form

$$
\boldsymbol{\Phi}_{\xi}\left(t-h_{\iota}\right)= \begin{cases}\mathbf{0}, & 0 \leq t<h_{\iota} \\ \mathbf{D}_{\xi \iota} \boldsymbol{\Phi}_{\xi}(t), & h_{\iota} \leq t \leq 1\end{cases}
$$

Since the parameter $\xi$ is arbitrarily selected, hence we have $h_{\iota} \xi^{k-1} \in \mathbb{N}$. We define

$$
n_{d_{\iota}}=h_{\iota} \xi^{k-1} \text {. }
$$


When $\frac{n-1}{\xi^{k-1}} \leq t-h_{\iota} \leq \frac{n}{\xi^{k-1}}$ we see that $\frac{n_{d_{\iota}}+n-1}{\xi^{k-1}} \leq t \leq \frac{n_{d_{\iota}}+n}{\xi^{k-1}}$, so by introducing a $M \times 1$ vector $\boldsymbol{\varphi}_{n}^{\xi}(t)$ as

$$
\boldsymbol{\varphi}_{n}^{\xi}(t)=\left[\phi_{n 0}^{\xi}(t), \phi_{n 1}^{\xi}(t), \phi_{n 2}^{\xi}(t), \ldots, \phi_{n M-1}^{\xi}(t)\right]^{\top}
$$

we can write

$$
\boldsymbol{\varphi}_{n}^{\xi}\left(t-h_{\iota}\right)=\mathbf{I}_{M} \boldsymbol{\varphi}_{n+n_{d_{\iota}} m}^{\xi}(t) .
$$

If $n>\xi^{k-1}-n_{d_{\iota}}$, then $\boldsymbol{\varphi}_{n}^{\xi}\left(t-h_{\iota}\right)=\mathbf{0}$. Thus the matrix $\mathbf{D}_{\xi \iota}$ is a square matrix of order $\xi^{k-1} M$ as follows

$$
\left.\mathbf{D}_{\xi \iota}=\left[\begin{array}{cccccccc}
\overbrace{\mathbf{0}} & \cdots & \mathbf{0} & \mathbf{I}_{M} & \mathbf{0} & \mathbf{0} & \cdots & \mathbf{0} \\
\mathbf{0} & \cdots & \mathbf{0} & \mathbf{0} & \mathbf{I}_{M} & \mathbf{0} & \cdots & \mathbf{0} \\
\mathbf{0} & \cdots & \mathbf{0} & \mathbf{0} & \mathbf{0} & \mathbf{I}_{M} & \cdots & \mathbf{0} \\
\vdots & \ddots & \vdots & \vdots & \vdots & \vdots & \ddots & \mathbf{0} \\
\mathbf{0} & \cdots & \mathbf{0} & \mathbf{0} & \mathbf{0} & \mathbf{0} & \cdots & \mathbf{I}_{M} \\
\mathbf{0} & \cdots & \mathbf{0} & \mathbf{0} & \mathbf{0} & \mathbf{0} & \cdots & \mathbf{0} \\
\vdots & \ddots & \vdots & \vdots & \vdots & \vdots & \ddots & \vdots \\
\mathbf{0} & \cdots & \mathbf{0} & \mathbf{0} & \mathbf{0} & \mathbf{0} & \mathbf{0} & \mathbf{0}
\end{array}\right]\right\} \xi^{k-1}-n_{d_{\iota}}
$$

and the right-hand side of this last expression can be written compactly in a more useful form as

$$
\mathbf{D}_{\xi \iota}=\left[\begin{array}{cc}
\mathbf{0}_{\left(\xi^{k-1}-n_{d_{\iota}}\right) M \times n_{d_{\iota}} M} & \mathbf{I}_{\left(\xi^{k-1}-n_{d_{\iota}}\right) M} \\
\hdashline \mathbf{0}_{n_{d_{\iota}} M \times \xi^{k-1} M} M
\end{array}\right] .
$$

\subsection{The inverse time operational matrix of Legendre wavelets}

A reverse time ASLW vector, $\boldsymbol{\Phi}_{\xi}(1-t)$ is given by

$$
\boldsymbol{\Phi}_{\xi}(1-t)=\Upsilon_{\xi} \boldsymbol{\Phi}_{\xi}(t), \quad 0 \leq t \leq 1
$$

where the inverse or reverse time operational matrix of ASLWs is introduced by $\boldsymbol{\Upsilon}_{\xi}$. According to (6) we have

$$
\phi_{n m}^{\xi}(1-t)=\sqrt{\xi^{k-1}} c_{m} P_{m}\left(2 \xi^{k-1}(1-t)-2 n+1\right), \quad \frac{n-1}{\xi^{k-1}} \leq 1-t \leq \frac{n}{\xi^{k-1}} .
$$

By introducing

$$
n_{s}=\xi^{k-1}-n+1,
$$

from $\frac{n-1}{\xi^{k-1}} \leq 1-t \leq \frac{n}{\xi^{k-1}}$ it follows that $\frac{n_{s}-1}{\xi^{k-1}} \leq t \leq \frac{n_{s}}{\xi^{k-1}}$. Hence by (5),

$$
\phi_{n m}^{\xi}(1-t)=(-1)^{m} \phi_{n_{s} m}^{\xi}(t) .
$$

So we conclude that

$$
\mathbf{\Upsilon}_{\xi}=\left[\begin{array}{cccc}
\mathbf{0} & \cdots & \mathbf{0} & \mathbf{Z} \\
\mathbf{0} & \cdots & \mathbf{Z} & \mathbf{0} \\
\vdots & . \cdot & \vdots & \vdots \\
\mathbf{Z} & \cdots & \mathbf{0} & \mathbf{0}
\end{array}\right], \mathbf{Z}=\left[\begin{array}{ccccc}
1 & 0 & \cdots & 0 & 0 \\
0 & -1 & \cdots & 0 & 0 \\
\vdots & \vdots & \ddots & \vdots & \vdots \\
0 & 0 & \cdots & (-1)^{M-2} & 0 \\
0 & 0 & \cdots & 0 & (-1)^{M-1}
\end{array}\right]
$$

where $\boldsymbol{\Upsilon}_{\xi}$ and $\mathbf{Z}$ are square matrices of order $\xi^{k-1} M$ and $M$, respectively. 


\subsection{The piecewise delay operational matrix of Legendre wavelets}

In this section, we derive a matrix introduced as the piecewise delay operational matrix of Legendre wavelets $\mathbf{D}_{\xi}^{t}$, to express terms having the piecewise constant delay $h(t)$ in terms of ASLWs. $h(t)$ is defined by

$$
h(t)=\left\{\begin{array}{cl}
h_{1}, & 0 \leq t \leq t_{1} \\
h_{2}, & t_{1} \leq t \leq t_{2} \\
\vdots & \vdots \\
h_{j}, & t_{j-1} \leq t \leq t_{j}
\end{array}\right.
$$

where $t_{j}=1$. If $t_{j} \neq 1$, we must set $t / t_{f} \rightarrow t$. We rewrite (26) in the form

$$
h(t)=\left\{\begin{array}{cl}
\frac{\lambda_{1}}{\omega}, & 0 \leq t \leq \frac{v_{1}}{\omega} \\
\frac{\lambda_{2}}{\omega}, & \frac{v_{1}}{\omega} \leq t \leq \frac{v_{2}}{\omega} \\
\vdots & \vdots \\
\frac{\lambda_{j}}{\omega}, & \frac{v_{j-1}}{\omega} \leq t \leq \frac{v_{j}}{\omega}
\end{array}\right.
$$

where for $i=1,2, \ldots, j$, we have $\lambda_{i}, v_{i}, \omega \in \mathbb{N}$. We select $\xi=\kappa \omega$, where $\kappa \in \mathbb{N}$. From (26) and (22),

$$
\boldsymbol{\Phi}_{\xi}(t-h(t))=\left\{\begin{array}{cl}
\boldsymbol{\Phi}_{\xi}\left(t-\frac{\lambda_{1}}{\omega}\right), & 0 \leq t \leq t_{1} \\
\boldsymbol{\Phi}_{\xi}\left(t-\frac{\lambda_{2}}{\omega}\right), & t_{1} \leq t \leq t_{2} \\
\vdots & \vdots \\
\boldsymbol{\Phi}_{\xi}\left(t-\frac{\lambda_{j}}{\omega}\right), & t_{j-1} \leq t \leq t_{j},
\end{array}=\left\{\begin{array}{cl}
\mathbf{D}_{\xi 1}^{t} \boldsymbol{\Phi}_{\xi}(t), & h_{1} \leq t \leq t_{1} \\
\mathbf{D}_{\xi 2}^{t} \boldsymbol{\Phi}_{\xi}(t), & h_{2} \leq t \leq t_{2} \\
\vdots & \vdots \\
\mathbf{D}_{\xi j}^{t} \boldsymbol{\Phi}_{\xi}(t), & h_{j} \leq t \leq t_{j} .
\end{array}\right.\right.
$$

Thus we can write

$$
\boldsymbol{\Phi}_{\xi}(t-h(t))=\sum_{i=1}^{j} \mathbf{D}_{\xi i}^{t} \boldsymbol{\Phi}_{\xi}(t) .
$$

Now we see how to find these piecewise delay matrices. Proceeding exactly as we did in [4], by taking

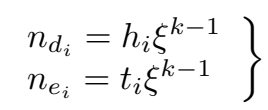

and

$$
\left.\begin{array}{l}
n_{\min _{i}}=n_{e_{i-1}}-n_{d_{i}} \\
n_{i}=n_{e_{i}}-n_{e_{i-1}}
\end{array}\right\}
$$

we define $\mathbf{D}_{\xi i}^{t}$ as

$$
\mathbf{D}_{\xi i}^{t}=\left[\begin{array}{c}
\mathbf{0}_{n_{m i n_{i}} M \times \xi^{k-1} M} \\
\boldsymbol{\Omega}_{i} \\
\mathbf{0}_{\left(\xi^{k-1}-n_{i}-n_{m i n_{i}}\right) M \times \xi^{k-1} M}
\end{array}\right]
$$

where

$$
\boldsymbol{\Omega}_{i}=\left[\begin{array}{lll}
\mathbf{0}_{n_{i} M \times n_{e_{i-1}} M} & \mathbf{I}_{n_{i} M} & \mathbf{0}_{n_{i} M \times\left(\xi^{k-1}-n_{e_{i}}\right) M}
\end{array}\right] .
$$

\section{Remark 1}

If $n_{m i n_{i}}<0$, we first set $n_{e_{i-1}}=n_{d_{i}}$, so $n_{\min _{i}}=0$. This is an exception, we only apply it to $\mathbf{D}_{\xi i}^{t}$. For more details and cases (may be needed in some problems), see Ref. [4]. 


\section{Optimal control and analysis of general linear delay systems via ASLWs}

In this section we are going to use the finding in the previous sections to the optimal control and analysis of general linear delay systems.

Consider a linear delay system containing delays and inverse time described by

$$
\begin{gathered}
\dot{\mathbf{x}}(t)=\mathbf{A}(t) \mathbf{x}(t)+\mathbf{B}(t) \mathbf{u}(t)+\sum_{\mu=1}^{a} \mathbf{E}_{\mu}(t) \mathbf{x}\left(t-h_{\mu}\right)+\sum_{\nu=1}^{b} \mathbf{F}_{\nu}(t) \mathbf{u}\left(t-h_{\nu}\right)+\mathbf{G}(t) \mathbf{x}\left(t_{f}-t\right)+\mathbf{H}(t) \mathbf{x}(t-h(t)), \\
\mathbf{x}(0)=\mathbf{x}_{0},\left\{\begin{array}{l}
\mathbf{x}(t)=\boldsymbol{\theta}(t), \quad-h_{x} \leq t<0 \\
\mathbf{u}(t)=\zeta(t),-h_{u} \leq t \leq 0,
\end{array}\right.
\end{gathered}
$$

where $t \in\left[0, t_{f}\right], \mathbf{x}(t) \in \mathbb{R}^{q}$ and $\mathbf{u}(t) \in \mathbb{R}^{r}$ are the state and control vectors, $\mathbf{A}(t), \mathbf{B}(t), \mathbf{E}_{\mu}(t), \mathbf{F}_{\nu}(t), \mathbf{G}(t)$, and $\mathbf{H}(t)$ are matrices of appropriate dimensions, $h_{\mu}$ and $h_{\nu}$ are delays, $h(t)$ is piecewise constant delay, $\mathbf{x}_{0}$ is the initial condition, $h_{x}$ is the supreme of $h_{\mu}$ and $h_{i}, h_{u}$ is the supreme of $h_{\nu}, \boldsymbol{\theta}(t) \in \mathbb{R}^{q}$ and $\zeta(t) \in \mathbb{R}^{r}$ are, respectively, initial state and initial control vector functions. The problem is to find the optimal controls and corresponding trajectories for the described system, which minimize the quadratic performance index

$$
J=\frac{1}{2} \mathbf{x}^{\top}\left(t_{f}\right) \mathbf{T} \mathbf{x}\left(t_{f}\right)+\frac{1}{2} \int_{0}^{t_{f}}\left\{\mathbf{x}^{\top}(t) \mathbf{Q}(t) \mathbf{x}(t)+\mathbf{u}^{\top}(t) \mathbf{R}(t) \mathbf{u}(t)\right\} d t,
$$

where $\mathbf{T}$ and $\mathbf{Q}(t)$ are symmetric, positive semi-definite matrices, $\mathbf{R}(t)$ is a symmetric, positive definite matrix.

Since Legendre wavelets are defined on $[0,1]$, we must change the range of the independent variable $t$ such that $0 \leq t \leq 1$; thus we set $t / t_{f} \rightarrow t, h_{\mu} / t_{f} \rightarrow h_{\mu}, h_{\nu} / t_{f} \rightarrow h_{\nu}$, and $h_{i} / t_{f} \rightarrow h_{i}$. We begin by defining $\xi$. In (27), we have assumed $h_{i}=\frac{\lambda_{i}}{\omega}$; we take $h_{\mu}=\frac{\epsilon_{\mu}}{\omega_{\mu}}, h_{\nu}=\frac{\epsilon_{\nu}}{\omega_{\nu}}$, where $\forall \mu=1, \ldots, a$ and $\forall \nu=1, \ldots, b$ we have $\epsilon_{\mu}, \omega_{\mu}, \epsilon_{\nu}, \omega_{\nu} \in \mathbb{N}$. Therefore we choose

$$
\xi=\kappa \cdot \operatorname{LCM}\left(\left\{\omega_{\mu}\right\},\left\{\omega_{\nu}\right\}, \omega\right),
$$

where $\kappa \in \mathbb{N}$. We select $\xi$ according to the true values of delays and this plays the key role in the construction of an accurate model of the time-delay system. Now we parameterize the state and control vectors by (9)-(11) as

$$
\left.\begin{array}{l}
\mathbf{x}(t)=\hat{\mathbf{\Phi}}_{\xi}^{\top}(t) \mathbf{X}_{\xi} \\
\mathbf{u}(t)=\check{\boldsymbol{\Phi}}_{\xi}^{\top}(t) \mathbf{U}_{\xi}
\end{array}\right\}
$$

where we define $\hat{.}=. \otimes \mathbf{I}_{q}, . . .=. . \otimes \mathbf{I}_{r}, \otimes$ is Kronecker product [28], $\mathbf{X}_{\xi}$ and $\mathbf{U}_{\xi}$ are $\xi^{k-1} q M \times 1$ and $\xi^{k-1} r M \times$ 1 column vectors of unknown parameters and

$$
\begin{aligned}
\mathbf{X}_{\xi} & =\left[X_{10}^{\xi 1}, \ldots, X_{10}^{\xi q}, \ldots, X_{1 M-1}^{\xi 1}, \ldots, X_{1 M-1}^{\xi q}, \ldots, X_{\xi^{k-1} M-1}^{\xi 1}, \ldots, X_{\xi^{k-1} M-1}^{\xi q}\right]^{\top}, \\
\mathbf{U}_{\xi} & =\left[U_{10}^{\xi 1}, \ldots, U_{10}^{\xi r}, \ldots, U_{1 M-1}^{\xi 1}, \ldots, U_{1 M-1}^{\xi r}, \ldots, U_{\xi^{k-1} M-1}^{\xi 1}, \ldots, U_{\xi^{k-1} M-1}^{\xi r}\right]^{\top} .
\end{aligned}
$$

We express the initial condition as

$$
\mathbf{x}_{0}=\hat{\boldsymbol{\Phi}}_{\xi}^{\top}(t) \mathbf{X}_{\xi}^{0},
$$

where $\mathbf{X}_{\xi}^{0}$ is a known $\xi^{k-1} q M \times 1$ column vector and

$$
\mathbf{X}_{\xi}^{0}=\frac{1}{\sqrt{\xi^{k-1}}}[\overbrace{\mathbf{X}^{\xi 0}, \mathbf{X}^{\xi 0}, \ldots, \mathbf{X}^{\xi 0}}^{\xi^{k-1} \text { times }}]^{\top}, \mathbf{X}^{\xi 0}=[\mathbf{x}_{0}^{\top}, \overbrace{0,0, \ldots, 0}^{q(M-1)}] .
$$

We express the matrices of the state equation in the terms of the new Legendre scaling functions as

$$
\mathbf{A}(t)=\mathbf{A}_{\xi} \hat{\boldsymbol{\Phi}}_{\xi}(t), \mathbf{B}(t)=\mathbf{B}_{\xi} \check{\boldsymbol{\Phi}}_{\xi}(t), \mathbf{E}_{\mu}(t)=\mathbf{E}_{\mu \xi} \hat{\boldsymbol{\Phi}}_{\xi}(t), \mathbf{F}_{\nu}(t)=\mathbf{F}_{\nu \xi} \check{\boldsymbol{\Phi}}_{\xi}(t), \mathbf{G}(t)=\mathbf{G}_{\xi} \hat{\boldsymbol{\Phi}}_{\xi}(t), \mathbf{H}(t)=\mathbf{H}_{\xi} \hat{\boldsymbol{\Phi}}_{\xi}(t) .
$$


For $\mu=1,2, \ldots, a$ and $\nu=1,2, \ldots, b$, we find $\left\{n_{d_{\mu}}\right\},\left\{n_{d_{\nu}}\right\}$ from (23). Then we expand the initial functions by

$$
\begin{aligned}
& \mathbf{x}\left(t-h_{\mu}\right)=\boldsymbol{\theta}\left(t-h_{\mu}\right)+\hat{\boldsymbol{\Phi}}_{\xi}^{\top}\left(t-h_{\mu}\right) \mathbf{X}_{\xi}=\hat{\boldsymbol{\Phi}}_{\xi}^{\top}(t) \boldsymbol{\theta}_{\mu \xi}+\hat{\boldsymbol{\Phi}}_{\xi}^{\top}(t) \hat{\mathbf{D}}_{\mu \xi}^{\top} \mathbf{X}_{\xi}, \\
& \mathbf{u}\left(t-h_{\nu}\right)=\zeta\left(t-h_{\nu}\right)+\check{\boldsymbol{\Phi}}_{\xi}^{\top}\left(t-h_{\nu}\right) \mathbf{U}_{\xi}=\check{\boldsymbol{\Phi}}_{\xi}^{\top}(t) \zeta_{\nu \xi}+\check{\boldsymbol{\Phi}}_{\xi}^{\top}(t) \check{\mathbf{D}}_{\nu \xi}^{\top} \mathbf{U}_{\xi},
\end{aligned}
$$

where each of $\mathbf{D}_{\mu \xi}$ and $\mathbf{D}_{\nu \xi}$ obtained by (24), $\theta_{\mu \xi}$ and $\zeta_{\nu \xi}$ are constant matrices which defined by

$$
\begin{gathered}
\theta_{\mu \xi}=[\theta_{10}^{\mu \xi 1}, \ldots, \theta_{10}^{\mu \xi q}, \ldots, \theta_{1 M-1}^{\mu \xi 1}, \ldots, \theta_{1 M-1}^{\mu \xi q}, \ldots, \theta_{n_{d_{\mu}} M-1}^{\mu \xi 1}, \ldots, \theta_{n_{d_{\mu}} M-1}^{\mu \xi q}, \overbrace{0,0,0, \ldots, 0}^{\left(\xi^{k-1}-n_{d_{\mu}}\right) q M}]^{\top}, \\
\zeta_{\nu \xi}=[\zeta_{10}^{\nu \xi 1}, \ldots, \zeta_{10}^{\nu \xi r}, \ldots, \zeta_{1 M-1}^{\nu \xi 1}, \ldots, \zeta_{1 M-1}^{\nu \xi r}, \ldots, \zeta_{n_{d_{\nu} M-1}}^{\nu \xi 1}, \ldots, \zeta_{n_{d_{\nu} M-1}}^{\nu \xi r}, \overbrace{0,0,0, \ldots, 0}^{\left(\xi^{k-1}-n_{d_{\nu}}\right) r M}]^{\top} .
\end{gathered}
$$

$\theta_{n m}^{\mu \xi \alpha}$ and $\zeta_{n m}^{\nu \xi \beta}$ in which $\alpha=1,2, \ldots, q$ and $\beta=1,2, \ldots, r$ can be obtained by (12).

\section{Remark 2}

It should be noted that the presented expansions of the integrals of delayed terms which were introduced in [6] and have been used in $[4,7]$, differ from the expansions presented in similar works for those integrals, that is, $\int_{0}^{t} \mathbf{E}_{\mu}\left(t^{\prime}\right) \mathbf{x}\left(t^{\prime}-h_{\mu}\right) d t^{\prime}$ and $\int_{0}^{t} \mathbf{F}_{\nu}\left(t^{\prime}\right) \mathbf{u}\left(t^{\prime}-h_{\nu}\right) d t^{\prime}$, for example, some of the literature are [29-32] and those listed in [6]. When we use our expansions, by calling $\hat{\mathbf{P}}_{\xi}^{\top} \tilde{\mathbf{E}}_{\mu \xi} \boldsymbol{\theta}_{\mu \xi}$ and $\hat{\mathbf{P}}_{\xi}^{\top} \tilde{\mathbf{F}}_{\nu \xi} \zeta_{\nu \xi}$, we can see (43) and (44) provide exact results and there is no need to define a matrix for integrating the desired wavelet vector from 0 to delay(s), that is, the constant matrix $\mathbf{Z}$ have been defined in the literature.

By setting

$$
\mathbf{D}_{\xi}^{t}=\sum_{i=1}^{j} \mathbf{D}_{\xi i}^{t}
$$

in $(28)$ and

$$
\boldsymbol{\theta}_{\xi}^{t}=\sum_{i=1}^{j} \boldsymbol{\theta}_{\xi i}^{t}
$$

in $\boldsymbol{\theta}(t-h(t))=\hat{\boldsymbol{\Phi}}_{\xi}^{\top}(t) \sum_{i=1}^{j} \boldsymbol{\theta}_{\xi i}^{t}$, we can write

$$
\mathbf{x}(t-h(t))=\boldsymbol{\theta}(t-h(t))+\hat{\boldsymbol{\Phi}}_{\xi}^{\top}(t-h(t)) \mathbf{X}_{\xi}=\hat{\boldsymbol{\Phi}}_{\xi}^{\top}(t) \boldsymbol{\theta}_{\xi}^{t}+\hat{\boldsymbol{\Phi}}_{\xi}^{\top}(t) \hat{\mathbf{D}}_{\xi}^{t^{\top}} \mathbf{X}_{\xi}
$$

Now we see how to compute the $\xi^{k-1} q M \times 1$ vector $\theta_{\xi}^{t}$. In the rescaled form of (26),

I when $h_{i} \leq t_{i-1}$, then we have

$$
\boldsymbol{\theta}_{\xi i}^{t}=\mathbf{0}
$$

II when $t_{i-1}<h_{i}<t_{i}$, we have

$$
\boldsymbol{\theta}_{\xi i}^{t}=[\overbrace{0,0, \ldots, 0}^{n_{e_{i-1}} q M}, \theta_{n_{e_{i-1}+1} 0}^{\xi i 1}, \ldots, \theta_{n_{e_{i-1}}+10}^{\xi i q}, \ldots, \theta_{n_{d_{i}} M-1}^{\xi i 1}, \ldots, \theta_{n_{d_{i}} M-1}^{\xi i q}, \overbrace{0,0,0, \ldots, 0}^{\left(\xi^{k-1}-n_{d_{i}}\right) q M}]^{\top} ;
$$

III otherwise, when $h_{i} \geq t_{i}$

$$
\boldsymbol{\theta}_{\xi i}^{t}=[\overbrace{0,0, \ldots, 0}^{n_{e_{i-1}} q M}, \theta_{n_{e_{i-1}}+10}^{\xi i 1}, \ldots, \theta_{n_{e_{i-1}}+1}^{\xi i q}, \ldots, \theta_{n_{e_{i}} M-1}^{\xi i 1}, \ldots, \theta_{n_{e_{i}} M-1}^{\xi i q}, \overbrace{0,0,0, \ldots, 0}^{\left(\xi^{k-1}-n_{e_{i}}\right) q M}]^{\top} .
$$


By integrating the rescaled equation of (33) from 0 to $t$, substituting the findings given in (37), (38), (40)-(42), (47) and using (25), we get

$$
\begin{aligned}
\hat{\boldsymbol{\Phi}}_{\xi}^{\top}(t) \mathbf{X}_{\xi}-\hat{\boldsymbol{\Phi}}_{\xi}^{\top}(t) \mathbf{X}_{\xi}^{0}= & t_{f} \int_{0}^{t}\left[\mathbf{A}_{\xi} \hat{\boldsymbol{\Phi}}_{\xi}(\vartheta) \hat{\boldsymbol{\Phi}}_{\xi}^{\top}(\vartheta) \mathbf{X}_{\xi}+\mathbf{B}_{\xi} \check{\boldsymbol{\Phi}}_{\xi}(\vartheta) \check{\boldsymbol{\Phi}}_{\xi}^{\top}(\vartheta) \mathbf{U}_{\xi}+\sum_{\mu=1}^{a}\left(\mathbf{E}_{\mu \xi} \hat{\boldsymbol{\Phi}}_{\xi}(\vartheta) \hat{\boldsymbol{\Phi}}_{\xi}^{\top}(\vartheta) \boldsymbol{\theta}_{\mu \xi}\right.\right. \\
& \left.+\mathbf{E}_{\mu \xi} \hat{\boldsymbol{\Phi}}_{\xi}(\vartheta) \hat{\boldsymbol{\Phi}}_{\xi}^{\top}(\vartheta) \hat{\mathbf{D}}_{\mu \xi}^{\top} \mathbf{X}_{\xi}\right)+\sum_{\nu=1}^{b}\left(\mathbf{F}_{\nu \xi} \check{\boldsymbol{\Phi}}_{\xi}(\vartheta) \check{\boldsymbol{\Phi}}_{\xi}^{\top}(\vartheta) \boldsymbol{\zeta}_{\nu \xi}+\mathbf{F}_{\nu \xi} \check{\boldsymbol{\Phi}}_{\xi}(\vartheta) \check{\boldsymbol{\Phi}}_{\xi}^{\top}(\vartheta) \check{\mathbf{D}}_{\nu \xi}^{\top} \mathbf{U}_{\xi}\right) \\
& \left.+\mathbf{G}_{\xi} \hat{\boldsymbol{\Phi}}_{\xi}(\vartheta) \hat{\boldsymbol{\Phi}}_{\xi}^{\top}(\vartheta) \hat{\boldsymbol{\Upsilon}}_{\xi}^{\top} \mathbf{X}_{\xi}+\mathbf{H}_{\xi} \hat{\boldsymbol{\Phi}}_{\xi}(\vartheta) \hat{\boldsymbol{\Phi}}_{\xi}^{\top}(\vartheta) \boldsymbol{\theta}_{\xi}^{t}+\mathbf{H}_{\xi} \hat{\boldsymbol{\Phi}}_{\xi}(\vartheta) \hat{\boldsymbol{\Phi}}_{\xi}^{\top}(\vartheta) \hat{\mathbf{D}}_{\xi}^{t^{\top}} \mathbf{X}_{\xi}\right] d \vartheta
\end{aligned}
$$

Now, by (17) and (13)

$$
\begin{aligned}
\hat{\mathbf{\Phi}}_{\xi}^{\top}(t)\left[\mathbf{X}_{\xi}-\mathbf{X}_{\xi}^{0}\right]= & t_{f} \hat{\mathbf{\Phi}}_{\xi}^{\top}(t)\left[\hat{\mathbf{P}}_{\xi}^{\top} \tilde{\mathbf{A}}_{\xi} \mathbf{X}_{\xi}+\hat{\mathbf{P}}_{\xi}^{\top} \tilde{\mathbf{B}}_{\xi} \mathbf{U}_{\xi}+\sum_{\mu=1}^{a}\left(\hat{\mathbf{P}}_{\xi}^{\top} \tilde{\mathbf{E}}_{\mu \xi} \boldsymbol{\theta}_{\mu \xi}+\hat{\mathbf{P}}_{\xi}^{\top} \tilde{\mathbf{E}}_{\mu \xi} \hat{\mathbf{D}}_{\mu \xi}^{\top} \mathbf{X}_{\xi}\right)\right. \\
& \left.+\sum_{\nu=1}^{b}\left(\hat{\mathbf{P}}_{\xi}^{\top} \tilde{\mathbf{F}}_{\nu \xi} \zeta_{\nu \xi}+\hat{\mathbf{P}}_{\xi}^{\top} \tilde{\mathbf{F}}_{\nu \xi} \check{\mathbf{D}}_{\nu \xi}^{\top} \mathbf{U}_{\xi}\right)+\hat{\mathbf{P}}_{\xi}^{\top} \tilde{\mathbf{G}}_{\xi} \hat{\mathbf{Y}}_{\xi}^{\top} \mathbf{X}_{\xi}+\hat{\mathbf{P}}_{\xi}^{\top} \tilde{\mathbf{H}}_{\xi} \boldsymbol{\theta}_{\xi}^{t}+\hat{\mathbf{P}}_{\xi}^{\top} \tilde{\mathbf{H}}_{\xi} \hat{\mathbf{D}}_{\xi}^{t^{\top}} \mathbf{X}_{\xi}\right]
\end{aligned}
$$

where each of the product operational matrices can be obtained from (19)-(21). In this definition of Legendre wavelets, the time interval $[0,1]$ is divided into $\xi^{k-1}$ subintervals. To ensure continuity of the state across these subintervals [14], we impose the following constraints at the boundary points $t_{\iota}$

$$
x_{\alpha}\left(t_{\iota}^{-}\right)=x_{\alpha}\left(t_{\iota}^{+}\right), \alpha=1,2, \ldots, q .
$$

By setting

we must have

$$
\boldsymbol{\varsigma}_{\iota}^{\xi}(t)=\boldsymbol{\varphi}_{\iota}^{\xi^{\top}}(t)
$$

$$
\boldsymbol{\varsigma}_{\iota}^{\xi}\left(t_{\iota}\right)\left[X_{\iota 0}^{\xi \alpha}, X_{\iota 1}^{\xi \alpha}, \ldots, X_{\iota M-1}^{\xi \alpha}\right]^{\top}-\boldsymbol{\varsigma}_{\iota+1}^{\xi}\left(t_{\iota}\right)\left[X_{\iota+10}^{\xi \alpha}, X_{\iota+11}^{\xi \alpha}, \ldots, X_{\iota+1 M-1}^{\xi \alpha}\right]^{\top}=0 .
$$

This yields, written in matrix form,

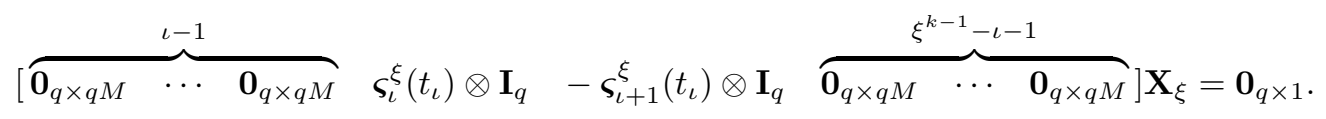

Knowing the maximum value of $\iota+1$ is $\xi^{k-1}$, we find $\iota=1,2, \ldots, \xi^{k-1}-1, t_{\iota}=\frac{\iota}{\xi^{k-1}}$ and the compatibility constraint can be expressed as

$$
\left(\left[\begin{array}{cccccc}
\boldsymbol{\varsigma}_{1}^{\xi}\left(t_{1}\right) & -\boldsymbol{\varsigma}_{2}^{\xi}\left(t_{1}\right) & \mathbf{0}_{1 \times M} & \cdots & \mathbf{0}_{1 \times M} & \mathbf{0}_{1 \times M} \\
\mathbf{0}_{1 \times M} & \boldsymbol{\varsigma}_{2}^{\xi}\left(t_{2}\right) & -\boldsymbol{\varsigma}_{3}^{\xi}\left(t_{2}\right) & \cdots & \mathbf{0}_{1 \times M} & \mathbf{0}_{1 \times M} \\
\vdots & \vdots & \vdots & \ddots & \vdots & \vdots \\
\mathbf{0}_{1 \times M} & \mathbf{0}_{1 \times M} & \mathbf{0}_{1 \times M} & \cdots & \boldsymbol{\varsigma}_{\xi^{k-1}-1}^{\xi}\left(t_{\xi^{k-1}-1}\right) & -\boldsymbol{\varsigma}_{\xi^{k-1}}^{\xi}\left(t_{\xi^{k-1}-1}\right)
\end{array}\right] \otimes \mathbf{I}_{q}\right) \mathbf{X}_{\xi}=\mathbf{0}
$$

Substituting $\mathbf{Q}(t)=\mathbf{Q}_{\xi} \hat{\boldsymbol{\Phi}}_{\xi}(t)$ and $\mathbf{R}(t)=\mathbf{R}_{\xi} \check{\boldsymbol{\Phi}}_{\xi}(t)$ in the performance index (35) and using (17), the properties of Kronecker product and (16), we can write

$$
\begin{aligned}
J & =\frac{1}{2} \mathbf{X}_{\xi}^{\top} \hat{\boldsymbol{\Phi}}_{\xi}(1) \mathbf{T} \hat{\boldsymbol{\Phi}}_{\xi}^{\top}(1) \mathbf{X}_{\xi}+\frac{1}{2} t_{f} \int_{0}^{1}\left(\mathbf{X}_{\xi}^{\top} \hat{\boldsymbol{\Phi}}_{\xi}(t) \mathbf{Q}_{\xi} \hat{\boldsymbol{\Phi}}_{\xi}(t) \hat{\boldsymbol{\Phi}}_{\xi}^{\top}(t) \mathbf{X}_{\xi}+\mathbf{U}_{\xi}^{\top} \check{\boldsymbol{\Phi}}_{\xi}(t) \mathbf{R}_{\xi} \check{\boldsymbol{\Phi}}_{\xi}(t) \check{\mathbf{\Phi}}_{\xi}^{\top}(t) \mathbf{U}_{\xi}\right) d t \\
& =\frac{1}{2} \mathbf{X}_{\xi}^{\top}\left(\boldsymbol{\Phi}_{\xi}(1) \boldsymbol{\Phi}_{\xi}^{\top}(1) \otimes \mathbf{T}\right) \mathbf{X}_{\xi}+\frac{1}{2} t_{f} \int_{0}^{1}\left(\mathbf{X}_{\xi}^{\top} \hat{\boldsymbol{\Phi}}_{\xi}(t) \hat{\boldsymbol{\Phi}}_{\xi}^{\top}(t) \tilde{\mathbf{Q}}_{\xi} \mathbf{X}_{\xi}+\mathbf{U}_{\xi}^{\top} \check{\boldsymbol{\Phi}}_{\xi}(t) \check{\boldsymbol{\Phi}}_{\xi}^{\top}(t) \tilde{\mathbf{R}}_{\xi} \mathbf{U}_{\xi}\right) d t \\
& =\frac{1}{2}\left\{\mathbf{X}_{\xi}^{\top}\left(\boldsymbol{\Phi}_{\xi}(1) \mathbf{\Phi}_{\xi}^{\top}(1) \otimes \mathbf{T}+t_{f} \tilde{\mathbf{Q}}_{\xi}\right) \mathbf{X}_{\xi}+\mathbf{U}_{\xi}^{\top}\left(t_{f} \tilde{\mathbf{R}}_{\xi}\right) \mathbf{U}_{\xi}\right\} .
\end{aligned}
$$


Thus from (48)-(50) we see the optimal control problem is converted to a simple optimization problem which is static in nature; the problem is

$$
\begin{aligned}
\min \frac{1}{2}\left[\begin{array}{c}
\mathbf{X}_{\xi} \\
\hdashline \mathbf{U}_{\xi}^{-}
\end{array}\right]^{\top}\left[\begin{array}{c:c}
\boldsymbol{\Xi}_{1} & \boldsymbol{\Xi}_{2} \\
\hdashline \mathbf{\Xi}_{3} & \mathbf{\Xi}_{4}
\end{array}\right]\left[\begin{array}{c}
\mathbf{X}_{\xi} \\
\hdashline \mathbf{U}_{\xi}^{-}
\end{array}\right] \\
\text {subject to } \quad\left[\begin{array}{c:c}
\boldsymbol{\Lambda}_{1} & \boldsymbol{\Lambda}_{2} \\
\hdashline \overline{\boldsymbol{\Lambda}}_{3}^{-} & \overline{\boldsymbol{\Lambda}}_{4}
\end{array}\right]\left[\begin{array}{c}
\mathbf{X}_{\xi} \\
\hdashline \overline{\mathbf{U}}_{\xi}^{-}
\end{array}\right]=\left[\begin{array}{c}
\boldsymbol{\Delta}_{1} \\
\hdashline \boldsymbol{\Delta}_{2}^{--}
\end{array}\right],
\end{aligned}
$$

where

$$
\begin{aligned}
& \boldsymbol{\Xi}_{1}=\boldsymbol{\Phi}_{\xi}(1) \boldsymbol{\Phi}_{\xi}^{\top}(1) \otimes \mathbf{T}+t_{f} \tilde{\mathbf{Q}}_{\xi}, \boldsymbol{\Xi}_{2}=\mathbf{0}_{q \varrho \times r \varrho}, \boldsymbol{\Xi}_{3}=\mathbf{0}_{r \varrho \times q \varrho}, \boldsymbol{\Xi}_{4}=t_{f} \tilde{\mathbf{R}}_{\xi}, \\
& \boldsymbol{\Lambda}_{1}=t_{f}\left(\hat{\mathbf{P}}_{\xi}^{\top} \tilde{\mathbf{A}}_{\xi}+\sum_{\mu=1}^{a} \hat{\mathbf{P}}_{\xi}^{\top} \tilde{\mathbf{E}}_{\mu \xi} \hat{\mathbf{D}}_{\mu \xi}^{\top}+\hat{\mathbf{P}}_{\xi}^{\top} \tilde{\mathbf{G}}_{\xi} \hat{\mathbf{Y}}_{\xi}^{\top}+\hat{\mathbf{P}}_{\xi}^{\top} \tilde{\mathbf{H}}_{\xi} \hat{\mathbf{D}}_{\xi}^{t^{\top}}\right)-\mathbf{I}_{q \varrho}, \boldsymbol{\Lambda}_{2}=t_{f}\left(\hat{\mathbf{P}}_{\xi}^{\top} \tilde{\mathbf{B}}_{\xi}+\sum_{\nu=1}^{b} \hat{\mathbf{P}}_{\xi}^{\top} \tilde{\mathbf{F}}_{\nu \xi} \check{\mathbf{D}}_{\nu \xi}^{\top}\right), \\
& \Lambda_{3}=\hat{\mathbf{\Psi}}_{\xi C} \\
& \boldsymbol{\Lambda}_{4}=\mathbf{0}_{\left(\xi^{k-1}-1\right) q \times r \varrho},
\end{aligned}
$$

in which $\varrho=\xi^{k-1} M$ and

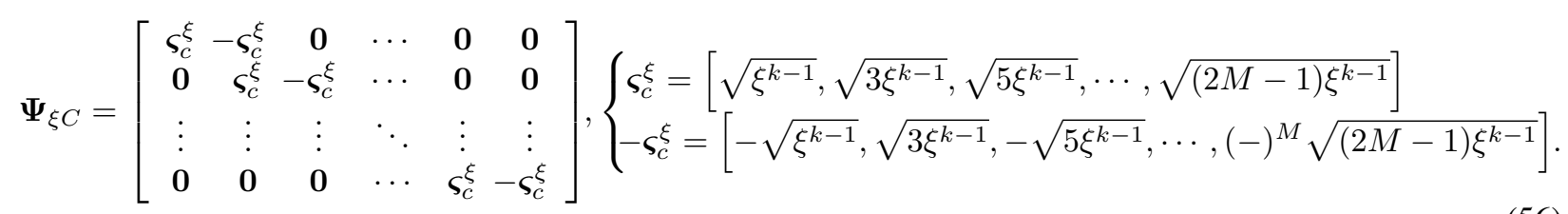

\section{Remark 3}

For a system described by (33)-(34) with time-invariant control weighted and state weighted matrices in the cost, where $J=\frac{1}{2} \mathbf{x}^{\top}\left(t_{f}\right) \mathbf{T} \mathbf{x}\left(t_{f}\right)+\frac{1}{2} \int_{0}^{t_{f}}\left\{\mathbf{x}^{\top}(t) \mathbf{Q} \mathbf{x}(t)+\mathbf{u}^{\top}(t) \mathbf{R u}(t)\right\} d t$, the matrices $\boldsymbol{\Xi}_{1}$ and $\boldsymbol{\Xi}_{4}$ in (53) become

$$
\boldsymbol{\Xi}_{1}=\boldsymbol{\Phi}_{\xi}(1) \boldsymbol{\Phi}_{\xi}^{\top}(1) \otimes \mathbf{T}+t_{f} \mathbf{I}_{\varrho} \otimes \mathbf{Q}, \quad \mathbf{\Xi}_{4}=t_{f} \mathbf{I}_{\varrho} \otimes \mathbf{R} .
$$

After selecting $\xi$ from (36), we must find (53)-(56) for the problem and then use the QP solver.

Now we introduce a further method to analyze systems with two kinds of delays. Consider a linear delay system containing reverse time described by

$$
\dot{\mathbf{x}}(t)=\mathbf{A}(t) \mathbf{x}(t)+\mathbf{B}(t) \mathbf{u}(t)+\mathbf{E}(t) \mathbf{x}\left(t-h_{\mu}\right)+\mathbf{F}(t) \mathbf{x}(t-h(t))+\mathbf{G}(t) \mathbf{x}\left(t_{f}-t\right), t \in\left[0, t_{f}\right]
$$

where the initial condition, the arbitrary function, and the input $\mathbf{u}(t)$ are

$$
\mathbf{x}(0)=\mathbf{x}_{0},\left\{\begin{array}{lr}
\mathbf{x}(t)=\boldsymbol{\theta}(t), & -h_{x} \leq t<0 \\
\mathbf{u}(t)=\mathbf{g}(t), & t \geq 0
\end{array}\right.
$$

The problem is to find $\mathbf{x}(t)$. Using a procedure similar to that discussed, we get

$$
\mathbf{X}_{\xi}-\mathbf{X}_{\xi}^{0}=t_{f}\left[\hat{\mathbf{P}}_{\xi}^{\top} \tilde{\mathbf{A}}_{\xi} \mathbf{X}_{\xi}+\hat{\mathbf{P}}_{\xi}^{\top} \tilde{\mathbf{B}}_{\xi} \mathbf{g}_{\xi}+\hat{\mathbf{P}}_{\xi}^{\top} \tilde{\mathbf{E}}_{\xi} \boldsymbol{\theta}_{\mu \xi}+\hat{\mathbf{P}}_{\xi}^{\top} \tilde{\mathbf{E}}_{\xi} \hat{\mathbf{D}}_{\mu \xi}^{\top} \mathbf{X}_{\xi}+\hat{\mathbf{P}}_{\xi}^{\top} \tilde{\mathbf{F}}_{\xi} \boldsymbol{\theta}_{\xi}^{t}+\hat{\mathbf{P}}_{\xi}^{\top} \tilde{\mathbf{F}}_{\xi} \hat{\mathbf{D}}_{\xi}^{t^{\top}} \mathbf{X}_{\xi}+\hat{\mathbf{P}}_{\xi}^{\top} \tilde{\mathbf{G}}_{\xi} \hat{\mathbf{\Upsilon}}_{\xi}^{\top} \mathbf{X}_{\xi}\right],
$$

where we have set

$$
\mathbf{g}(t)=\check{\mathbf{\Phi}}_{\xi}^{\top}(t) \mathbf{g}_{\xi}
$$

$\mathbf{g}_{\xi}$ is defined as follow

$$
\mathbf{g}_{\xi}=\left[g_{10}^{\xi 1}, \ldots, g_{10}^{\xi r}, \ldots, g_{1 M-1}^{\xi 1}, \ldots, g_{1 M-1}^{\xi r}, \ldots, g_{\xi^{k-1} M-1}^{\xi 1}, \ldots, g_{\xi^{k-1} M-1}^{\xi r}\right]^{\top} .
$$


Thus from (59), the response of the system expressed by (57) and (58) is (in terms of ASLWs)

$$
\begin{aligned}
\mathbf{X}_{\xi}=\left[\mathbf{I}_{q \varrho}-t_{f}\left(\hat{\mathbf{P}}_{\xi}^{\top} \tilde{\mathbf{A}}_{\xi}+\hat{\mathbf{P}}_{\xi}^{\top} \tilde{\mathbf{E}}_{\xi} \hat{\mathbf{D}}_{\mu \xi}^{\top}+\hat{\mathbf{P}}_{\xi}^{\top} \tilde{\mathbf{F}}_{\xi} \hat{\mathbf{D}}_{\xi}^{t^{\top}}+\hat{\mathbf{P}}_{\xi}^{\top} \tilde{\mathbf{G}}_{\xi} \hat{\mathbf{\Upsilon}}_{\xi}^{\top}\right)\right]^{-1} & {\left[\mathbf{X}_{\xi}^{0}+t_{f}\left(\hat{\mathbf{P}}_{\xi}^{\top} \tilde{\mathbf{B}}_{\xi} \mathbf{g}_{\xi}+\hat{\mathbf{P}}_{\xi}^{\top} \tilde{\mathbf{E}}_{\xi} \boldsymbol{\theta}_{\mu \xi}+\hat{\mathbf{P}}_{\xi}^{\top} \tilde{\mathbf{F}}_{\xi} \boldsymbol{\theta}_{\xi}^{t}\right)\right] . }
\end{aligned}
$$

To determine whether ASLW methods provide the accurate solutions and to see how to use ASLW methods in the case in which some matrices are time-invariant, we must use two given algorithms in [4] with some modifications (including Remark 3) which give ASLW methods in detailed steps suitable for implementation. Also by using the time-partition technique, as we did in this Ref., we can apply ASLWs on time-varying time-delay systems in which delays are time-varying.

\section{Numerical examples}

\subsection{Example 1}

We are interested in finding the optimal control and state which when applied to a TD system expressed by

$$
\begin{gathered}
\dot{x}(t)=-x(t)+x\left(t-\frac{1}{3}\right)+u(t)-0.5 u\left(t-\frac{2}{3}\right), \quad 0 \leq t \leq 1 \\
x(t)=1, \quad-\frac{1}{3} \leq t \leq 0 \\
u(t)=0, \quad-\frac{2}{3} \leq t \leq 0
\end{gathered}
$$

give an optimal cost $J^{*}$ described by

$$
J=\frac{1}{2} \int_{0}^{1}\left\{x^{2}(t)+\frac{1}{2} u^{2}(t)\right\} d t .
$$

According to the value of time delays, we select $\xi=3$. Choosing $k=2$ gives $n_{d_{\mu}}=1$ and $n_{d_{\nu}}=2$; then by $M=3$, from (15), (24), (39), (43)

$$
\begin{gathered}
\mathbf{P}_{\xi}=\frac{1}{6}\left[\begin{array}{ccc}
\mathbf{Y} & \mathbf{J} & \mathbf{J} \\
\mathbf{0} & \mathbf{Y} & \mathbf{J} \\
\mathbf{0} & \mathbf{0} & \mathbf{Y}
\end{array}\right], \mathbf{D}_{\mu \xi}=\left[\begin{array}{cc}
\mathbf{0}_{6 \times 3} & \mathbf{I}_{6} \\
\mathbf{0}_{3 \times 9}
\end{array}\right], \mathbf{D}_{\nu \xi}=\left[\begin{array}{cc}
\mathbf{0}_{3 \times 6} & \mathbf{I}_{3} \\
\mathbf{0}_{6 \times 9}
\end{array}\right], \\
\mathbf{X}_{\xi}^{0}=\frac{1}{\sqrt{3}}[1,0,0,1,0,0,1,0,0]^{\top}, \boldsymbol{\theta}_{\mu \xi}=\left[\frac{1}{\sqrt{3}}, 0,0,0,0,0,0,0,0\right]^{\top} .
\end{gathered}
$$

Constructing (51) and (52) and calling quadprog in MATLAB, we solve the QP problem and find

$$
x^{*}(t)=\left\{\begin{array}{ll}
0.734 t^{2}-0.947 t+0.999, & 0 \leq t \leq \frac{1}{3} \\
0.36 t^{2}-0.735 t+0.968, & \frac{1}{3} \leq t \leq \frac{2}{3} \\
0.0798 t^{2}+0.0943 t+0.541, & \frac{2}{3} \leq t \leq 1,
\end{array} \quad u^{*}(t)= \begin{cases}-0.968 t^{2}+1.09 t-0.986, & 0 \leq t \leq \frac{1}{3} \\
-0.396 t^{2}+1.44 t-1.17, & \frac{1}{3} \leq t \leq \frac{2}{3} \\
0.782 t^{2}-0.159 t-0.624, & \frac{2}{3} \leq t \leq 1\end{cases}\right.
$$

Also we use CLWs to solve this problem; we do this to compare the values of $J^{*}$. So, the comparisons are listed in Table 1 and show that by ASLWs, we can obtain more accurate results with low $k$. We see the obtained values of $\Delta J^{*}$ by ASLWs are very small. The results demonstrate that the proposed Legendre wavelet method converges rapidly and clearly show the improved accuracy. Obviously, by choosing $k=2$ in CLW method we cannot solve the problem. The optimal state and control are given in Figure 1 and we see the obvious boundary points are $t=1 / 3$ and $t=2 / 3$ which are exactly equal to the delays. In CLW method, we have used $k=6$ to find acceptable results which means we obtained the solutions as the piecewise defined functions containing 32 intervals. In Figure 1, we can see the fact that in CLW method we have to shift the delays. Up to these points we have motivated to apply Legendre wavelets with scaling in time-delay systems. As was mentioned in Remark 2, here, we can verify (43) by evaluating $\mathbf{P}_{\xi}^{\top} \boldsymbol{\theta}_{\mu \xi}$. 
Table 1. Comparison of $J^{*}$ in Example 1.

\begin{tabular}{ll}
\hline Method & Optimal cost $J^{*}$ \\
\hline ASCWs, $\xi=3, k=3, M=7$ [4] & 0.373112935279 \\
\hline CLWs, $k=2$ & not available \\
CLWs, $k=3, M=4$ & 0.35949955 \\
CLWs, $k=4, M=4$ & 0.37975995 \\
CLWs, $k=6, M=4$ & 0.37478424 \\
ASLWs, $\xi=3, k=2, M=3$ & 0.373112918644 \\
ASLWs, $\xi=3, k=2, M=4$ & 0.373112935296 \\
ASLWs, $\xi=3, k=3, M=3$ & 0.373112935274 \\
ASLWs, $\xi=3, k=2, M=7$ & 0.373112935279 \\
\hline
\end{tabular}
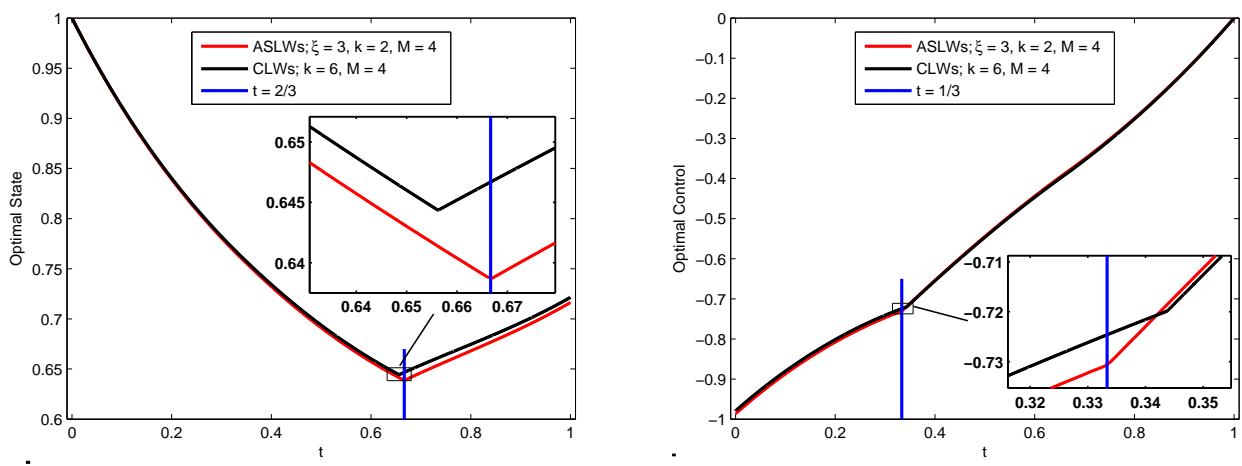

Figure 1. Comparison of optimal states and controls for Example 1.

\subsection{Example 2}

Given a linear, time-varying system

$$
\begin{gathered}
\dot{\mathbf{x}}(t)=\left[\begin{array}{ccc}
t & 1 & t^{2}+1 \\
1 & t & 0 \\
t^{2}+1 & 0 & t
\end{array}\right] \mathbf{x}\left(t-\frac{1}{3}\right)+\left[\begin{array}{cc}
1 & t+1 \\
t+1 & 1 \\
1 & 1
\end{array}\right] \mathbf{u}(t)+\left[\begin{array}{cc}
1 & t+1 \\
t & 1 \\
1 & t^{2}+1
\end{array}\right] \mathbf{u}\left(t-\frac{2}{3}\right)+\left[\begin{array}{ccc}
t^{2} & 1 & 1 \\
1 & 1 & 0 \\
t & 0 & 1
\end{array}\right] \mathbf{x}(1-t), \\
\mathbf{x}(t)=\left[\begin{array}{lll}
1 & 1 & 1
\end{array}\right]^{\top},-\frac{1}{3} \leq t \leq 0 \\
\mathbf{u}(t)=\left[\begin{array}{ll}
1 & 1
\end{array}\right]^{\top},-\frac{2}{3} \leq t \leq 0
\end{gathered}
$$

and the performance index

$$
J=\frac{1}{2} \int_{0}^{1}\left\{\mathbf{x}^{\top}(t)\left[\begin{array}{ccc}
1 & t & 0 \\
t & t^{2} & 0 \\
0 & 0 & t^{2}
\end{array}\right] \mathbf{x}(t)+\mathbf{u}^{\top}(t) \mathbf{I}_{2} \mathbf{u}(t)\right\} d t
$$

find the optimal cost.

By selecting $\xi=3,6, k=2$ and $M=5,7$, the corresponding results are reported in Table 2 and the superiority of ASLW method over CLW method is clear. As can been seen, with low order of approximation, the significant accuracy obtained and in applying ASLWs we have three options to increase the accuracy of the solution. 
Table 2. Comparison of numerical results in Example 2.

\begin{tabular}{ll}
\hline Method & $J^{*}$ \\
\hline CCWs, $k=7, M=5[6]$ & 3.1339565957 \\
ASCWs, $\xi=6, k=2, M=7[4]$ & 3.1083548837 \\
\hline CLWs, $k=2$ & not available \\
ASLWs, $\xi=3, k=2, M=5$ & 3.1083977271 \\
ASLWs, $\xi=3, k=2, M=7$ & 3.1083549039 \\
ASLWs, $\xi=6, k=2, M=7$ & 3.1083548870 \\
\hline
\end{tabular}

\subsection{Example 3}

We want to analyze the piecewise constant delay system described [5, p. 150]

$$
\begin{gathered}
\dot{x}(t)=x(t-h(t))+u(t) \\
x(t)=0, \quad t \leq 0 \\
u(t)=1, \quad t>0
\end{gathered}
$$

where

$$
h(t)= \begin{cases}0.1, & 0 \leq t \leq 0.35 \\ 0.3, & 0.35 \leq t \leq 0.7 \\ 0.5, & 0.7 \leq t \leq 1\end{cases}
$$

Using (27), we can write

$$
h(t)= \begin{cases}\frac{2}{20}, & 0 \leq t \leq \frac{7}{20} \\ \frac{6}{20}, & \frac{7}{20} \leq t \leq \frac{14}{20} \\ \frac{10}{20}, & \frac{14}{20} \leq t \leq \frac{20}{20}\end{cases}
$$

Hence we select $\xi=20$. By choosing $k=2$, from (29) and (30) we see $n_{0}=0$ and $n_{d}=2$ and $n_{\min _{1}}=-2$. According to Remark 1 , we set $n_{e_{0}}=2$, so $n_{\min _{1}}=0$ and $n_{1}=5$. Now from (31) and (32)

$$
\mathbf{D}_{\xi 1}^{t}=\left[\begin{array}{c}
\boldsymbol{\Omega}_{1} \\
\mathbf{0}_{15 M \times 20 M}
\end{array}\right], \boldsymbol{\Omega}_{1}=\left[\begin{array}{lll}
\mathbf{0}_{5 M \times 2 M} & \mathbf{I}_{5 M} & \mathbf{0}_{5 M \times 13 M}
\end{array}\right] .
$$

In like manner, we find

$$
\mathbf{D}_{\xi 2}^{t}=\left[\begin{array}{c}
\mathbf{0}_{M \times 20 M} \\
\boldsymbol{\Omega}_{2} \\
\mathbf{0}_{12 M \times 20 M}
\end{array}\right], \boldsymbol{\Omega}_{2}=\left[\begin{array}{lll}
\mathbf{0}_{7 M \times 7 M} & \mathbf{I}_{7 M} & \mathbf{0}_{7 M \times 6 M}
\end{array}\right]
$$

and

$$
\mathbf{D}_{\xi 3}^{t}=\left[\begin{array}{c}
\mathbf{0}_{4 M \times 20 M} \\
\boldsymbol{\Omega}_{3} \\
\mathbf{0}_{10 M \times 20 M}
\end{array}\right], \boldsymbol{\Omega}_{3}=\left[\begin{array}{ll}
\mathbf{0}_{6 M \times 14 M} & \mathbf{I}_{6 M}
\end{array}\right] .
$$

(45) gives $\mathbf{D}_{\xi}^{t}=\sum_{i=1}^{3} \mathbf{D}_{\xi i}^{t}$; from (60), by expressing $g(t)=1$ in terms of 7th-degree partial sum of ASLWs expansion, constructing $\mathbf{P}_{\xi}$ and using (61), we solve the problem. Obtaining the exact response of (63) by using CLWs is not possible, whereas we can do this by the use of ASLWs. Figure 2 shows the results of the arbitrary scaled Legendre and Chebyshev wavelets with $\xi=20, k=2$ and $M=8$. 


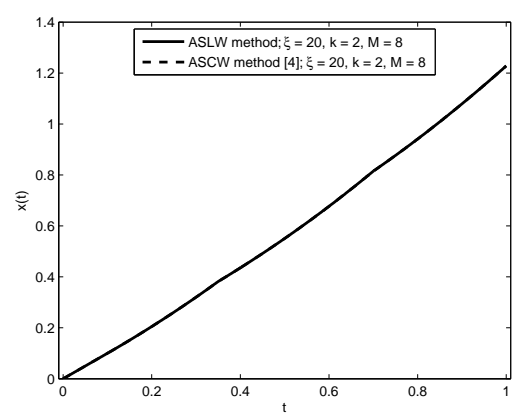

Figure 2. Response of (63).

\subsection{Example 4}

Obtain a solution to the state equation [5]

$$
\dot{x}(t)=-5 x(t)-5 x(t-h(t))+2 u(t)
$$

subject to the conditions

$$
\begin{aligned}
h(t)= & \begin{cases}0, & 0 \leq t \leq 0.8 \\
0.3, & 0.8 \leq t \leq 1.4 \\
0.6, & 1.4 \leq t \leq 1.7 \\
0.9, & 1.7 \leq t \leq 2\end{cases} \\
& x(t)=1, \quad t \leq 0 \\
& u(t)=1, t \geq 0
\end{aligned}
$$

We set $t / 2 \rightarrow t$, thus we have

Selecting $\xi=20, k=2$ and $M=8$, we get

$$
h(t)= \begin{cases}0, & 0 \leq t \leq \frac{8}{20} \\ \frac{3}{20}, & \frac{8}{20} \leq t \leq \frac{14}{20} \\ \frac{6}{20}, & \frac{14}{20} \leq t \leq \frac{17}{20} \\ \frac{9}{20}, & \frac{17}{20} \leq t \leq \frac{20}{20}\end{cases}
$$

$$
\begin{gathered}
\mathbf{D}_{\xi 1}^{t}=\left[\begin{array}{c}
\boldsymbol{\Omega}_{1} \\
\mathbf{0}_{96 \times 160}
\end{array}\right], \boldsymbol{\Omega}_{1}=\left[\begin{array}{ll}
\mathbf{I}_{64} & \mathbf{0}_{64 \times 96}
\end{array}\right], \\
\mathbf{D}_{\xi 2}^{t}=\left[\begin{array}{c}
\mathbf{0}_{40 \times 160} \\
\boldsymbol{\Omega}_{2} \\
\mathbf{0}_{72 \times 160}
\end{array}\right], \boldsymbol{\Omega}_{2}=\left[\begin{array}{lll}
\mathbf{0}_{48 \times 64} & \mathbf{I}_{48} & \mathbf{0}_{48 \times 48}
\end{array}\right], \\
\mathbf{D}_{\xi 3}^{t}=\left[\begin{array}{c}
\mathbf{0}_{64 \times 160} \\
\boldsymbol{\Omega}_{3} \\
\mathbf{0}_{72 \times 160}
\end{array}\right], \boldsymbol{\Omega}_{3}=\left[\begin{array}{lll}
\mathbf{0}_{24 \times 112} & \mathbf{I}_{24} & \mathbf{0}_{24 \times 24}
\end{array}\right]
\end{gathered}
$$

and

$$
\mathbf{D}_{\xi 4}^{t}=\left[\begin{array}{c}
\mathbf{0}_{64 \times 160} \\
\boldsymbol{\Omega}_{4} \\
\mathbf{0}_{72 \times 160}
\end{array}\right], \boldsymbol{\Omega}_{4}=\left[\begin{array}{ll}
\mathbf{0}_{24 \times 136} & \mathbf{I}_{24}
\end{array}\right] ;
$$

then, from (45) we have $\mathbf{D}_{\xi}^{t}=\sum_{i=1}^{4} \mathbf{D}_{\xi i}^{t}$. It follows from the discussion mentioned in Section 3 that for $i=1,2,3,4$ we see $\boldsymbol{\theta}_{\xi i}^{t}=\mathbf{0}$, Thus, from (46) we have $\boldsymbol{\theta}_{\xi}^{t}=\mathbf{0}$. Using (61), we solve the problem and obtain the response of the system. The exact solution of this piecewise constant delay system is presented in [5, p. 154]. A comparison of the results is given in Figure 3. 


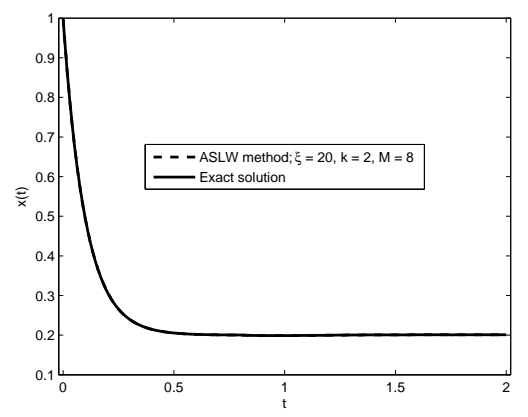

Figure 3. Comparison of responses of (64).

\subsection{Example 5}

Motivated by the fact that real-world systems have some imposed constraints, for example see [33,34], we consider this delay system in which different constraints are studied. It is desired to minimize the performance index which is given in (62) subject to the conditions

$$
\begin{aligned}
& \dot{\mathbf{x}}(t)=\left[\begin{array}{ccc}
t & 1 & 0 \\
0 & 1 & 0 \\
0 & 0 & t^{2}
\end{array}\right] \mathbf{x}(t)+\left[\begin{array}{cc}
1 & t+1 \\
t+1 & 1 \\
1 & 1
\end{array}\right] \mathbf{u}(t)+\left[\begin{array}{cc}
1 & t+1 \\
t & 1 \\
1 & t^{2}+1
\end{array}\right] \mathbf{u}\left(t-\frac{2}{5}\right) \\
& +\left[\begin{array}{ccc}
t^{2} & 1 & 1 \\
1 & 1 & 0 \\
t & 0 & 1
\end{array}\right] \mathbf{x}(1-t)+\left[\begin{array}{ccc}
t & 1 & t^{2}+1 \\
1 & t & 0 \\
t^{2}+1 & 0 & t
\end{array}\right] \mathbf{x}(t-h(t)), \quad 0 \leq t \leq 1 \\
& h(t)= \begin{cases}\frac{1}{5}, & 0 \leq t \leq \frac{1}{5} \\
\frac{2}{5}, & \frac{1}{5} \leq t \leq \frac{3}{5} \\
\frac{4}{5}, & \frac{3}{5} \leq t \leq 1\end{cases} \\
& \mathbf{x}(t)=\left[\begin{array}{lll}
t^{2}+1 & t^{2}+1 & t^{2}+1
\end{array}\right]^{\top}, \quad t \leq 0 \\
& \mathbf{u}(t)=\left[\begin{array}{ll}
t+1 & t+1
\end{array}\right]^{\top}, \quad-\frac{2}{5} \leq t \leq 0
\end{aligned}
$$

and

1. assume that the control and state are unconstrained.

2. let the final state constraint be

$$
x_{1}\left(t_{f}\right) \geq 0.6 \text { and } x_{2}\left(t_{f}\right) \leq-0.6 .
$$

3. let the restrictions in the control variables be

$$
\forall t \in\left[0, \frac{4}{5}\right],-1.9 \leq u_{1}(t) \leq-0.5 \text { and }-1.95 \leq u_{2}(t) \leq-0.4 .
$$

4. let the piecewise combined constraint be

$$
\begin{gathered}
\forall t \in\left[0, \frac{2}{5}\right], t^{2} x_{1}(t)-x_{2}(t)-t x_{3}(t)-u_{1}(t)+t u_{2}(t) \leq 0, \\
\forall t \in\left[\frac{3}{5}, 1\right], x_{1}(t) \geq 0.2 \text { and } x_{1}(t)-2 x_{2}(t)+x_{3}(t) \leq 0 .
\end{gathered}
$$

Obviously, solving the problem by applying CLW method is not possible. In this problem, we choose $\xi=5$. By setting $k=2$ and $M=7$ and using the given findings, Figure 4 shows the optimal states and controls obtained by the present method. In cases $1-4$, we find, in turn, $J^{*}=1.7554, J^{*}=1.8794, J^{*}=1.7927$, and $J^{*}=2.1766$. 

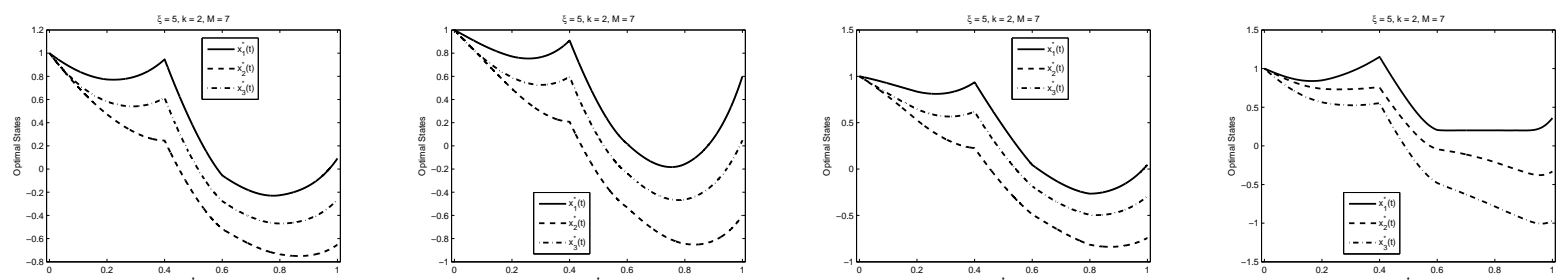

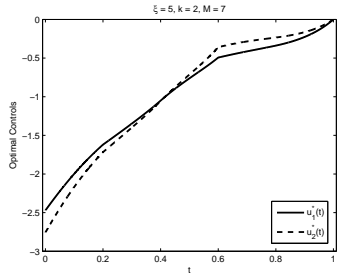

(a) case 1

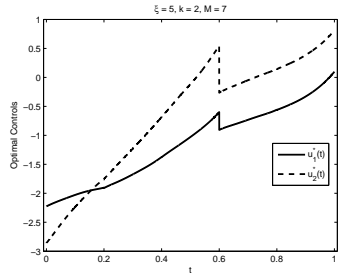

(b) case 2

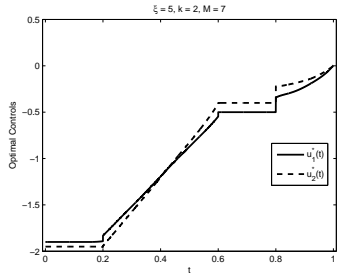

(c) case 3

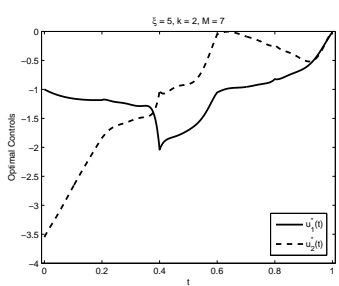

(d) case 4

Figure 4. Optimal states and controls for Example 5.

\section{Conclusion}

In the study of delay differential equations describing dynamical processes, Legendre wavelet method has a much wider range of capabilities than Legendre polynomial method. By using the conventional Legendre wavelet method, we may not be able to find accurate solutions of delay differential equations. Furthermore, we obviously cannot apply it on systems with piecewise constant delays. Hence, to achieve some improvement in accuracy and to develop the applicability of Legendre wavelet method, a flexible definition has been proposed in which the scaling parameter is selected according to the true values of delay. We saw that ASLW method can provide acceptable solutions with minimum number of subintervals, where its solution process is simpler than CLW method and considerable savings in computation time and memory are attained; also to improve the accuracy, we can increase the value of $\xi$ instead of $k$. By using the given concepts, ASLW method have been developed for the efficient analysis and optimal control of systems with piecewise constant delays and zero or non-zero initial functions.

\section{Acknowledgement}

I would like to thank Professor David G. Yu for the helpful discussions.

\section{REFERENCES}

1. A. Graps, An Introduction to Wavelets, IEEE computational science and engineering, vol. 2, no. 2, pp. 50-61, 1995.

2. E. Kreyszig, Advanced engineering mathematics, John Wiley \& Sons, 2010.

3. L.C. Andrews, Special functions of mathematics for engineers, New York: McGraw-Hill, 1992.

4. I. Malmir, Novel Chebyshev wavelets algorithms for optimal control and analysis of general linear delay models, Applied Mathematical Modelling, 2018. doi: https://doi.org/10.1016/j.apm.2018.12.009

5. K.B. Datta, B.M. Mohan, Orthogonal functions in systems and control, Advanced Series in Electrical and Computer Engineering, World Scientific Publishing Co., 1995.

6. I. Malmir, Optimal control of linear time-varying systems with state and input delays by Chebyshev wavelets, Statistics, Optimization \& Information Computing, vol. 5, no. 4, pp. 302-324, 2017.

7. I. Malmir, A novel wavelet-based optimal linear quadratic tracker for time-varying systems with multiple delays, arXiv preprint, arXiv: 1802.05618, 2018.

8. H.R. Sharif, M.A. Vali, M. Samavat, A.A. Gharavisi, A New Algorithm for Optimal Control of Time-Delay Systems, Applied Mathematical Sciences, vol. 5, no. 12, pp. 595-606, 2011.

9. W.A. Al-Salam, On the product of two Legendre polynomials, Mathematica Scandinavica, vol. 4, pp. 239-242, 1957. 
10. Y. Shen, W. Lin, Collocation method for the natural boundary integral equation, Applied Mathematics Letters, vol. 19, no. 11, pp. 1278-1285, 2006.

11. M. Razzaghi, S. Yousefi, Legendre wavelets direct method for variational problems, Mathematics and computers in simulation, vol. 53, no. 3, pp. 185-192, 2000.

12. M. Razzaghi, S. Oppenheimer, F. Ahmad, A Legendre wavelet method for the radiative transfer equation in remote sensing, Journal of electromagnetic waves and applications, vol. 16, no. 12, pp. 1681-1693, 2002.

13. K. Maleknejad, M.T. Kajani, Y. Mahmoudi, Numerical solution of linear Fredholm and Volterra integral equations of the second kind by using Legendre wavelets, Kybernetes, vol. 32, no. 9/10, pp. 1530-1539, 2003.

14. H. Jaddu, Optimal control of time-varying linear systems using wavelets, PhD, Japan Advanced Institute of Science and Technology, Ishikawa, Japan, 2006.

15. S. Ma, H. Gao, G. Zhang, L. Wu, Abel inversion using Legendre wavelets expansion, Journal of Quantitative Spectroscopy \& Radiative Transfer, vol. 107, no. 1, pp. 61-71, 2007.

16. R. Ebrahimi, M.A. Vali, M. Samavat, A.A. Gharavisi, A computational method for solving optimal control of singular systems using the Legendre wavelets, ICGST Automatic Control and System Engineering Journal, vol. 9, no. 2, pp. 1-6, 2009.

17. N. Liu, E.B. Lin, Legendre wavelet method for numerical solutions of partial differential equations, Numerical Methods for Partial Differential Equations, vol. 26, no. 1, pp. 81-94, 2010.

18. E. A. Rawashdeh, Legendre wavelets method for fractional integro-differential equations, Applied Mathematical Sciences, vol. 5, no. 2, pp. 2467-74, 2011.

19. C. Bandt, M. Barnsley, R. Devaney, K.J. Falconer, V. Kannan, V. Kumar P.B. (Editors), Fractals, Wavelets, and their Applications: Contributions from the International Conference and Workshop on Fractals and Wavelets, Springer, vol. 92, pp. 491-494, 2014.

20. A. Setia, B. Prakash, A.S. Vatsala, Numerical Solution by Fourth Order Fractional Integro-Differential Equation by Using Legendre Wavelets, Neural, Parallel, and Scientific Computations, vol. 23, pp. 377-386, 2015.

21. K. Maleknejad, E. Saeedipoor, R. Dehbozorgi, Legendre Wavelets Direct Method for the Numerical Solution of Fredholm Integral Equation of the First Kind, Proceedings of the World Congress on Engineering, vol.1, 2016.

22. S.T. Mohyud-din, M.A. Iqbal, U. Khan U, X.J. Yang, MHD squeezing flow between two parallel disks with suction or injection via Legendre wavelet-quasilinearization technique, Engineering Computations, vol. 34, no. 3, pp. 892-901, 2017.

23. S. Yadav, S. Upadhyay, K.N. Rai, Legendre Wavelet Modified Petrov-Galerkin Method in Two-Dimensional Moving Boundary Problem, Zeitschrift für Naturforschung A., vol. 73, no. 1, pp. 23-34, 2017.

24. S. Balaji, G. Hariharan, A novel wavelet approximation method for the solution of nonlinear differential equations with variable coefficients arising in astrophysics, Astrophysics and Space Science, vol. 363, no. 1, pp. 1-16, 2018.

25. S. Singh, V.K. Patel, V. K. Singh, Application of wavelet collocation method for hyperbolic partial differential equations via matrices, Applied Mathematics and Computation, vol. 320, pp. 407-24, 2018.

26. M. Sohaib, S. Haq, S. Mukhtar, I. Khan, Numerical solution of sixth-order boundary-value problems using Legendre wavelet collocation method, Results in physics, vol. 8, pp. 1204-1208, 2018.

27. M. Kumar, S. Upadhyay, K.N. Rai, A study of cryosurgery of lung cancer using Modified Legendre wavelet Galerkin method, Journal of thermal biology vol. 78, pp. 356-366, 2018.

28. Z. Lin, L. He, T. Wu, C. Xu, The parameter estimation of the multivariate matrix regression models, Statistics, Optimization \& Information Computing, vol. 6, no. 2, pp. 286-291, 2018.

29. H.R. Marzban, M. Razzaghi, Solution of time-varying delay systems by hybrid functions, Mathematics and Computers in Simulation, vol. 64, no. 6, pp. 597-607, 2004.

30. F. Khellat, N. Vasegh, Suboptimal control of linear systems with delays in state and input by orthonormal basis, International Journal of Computer Mathematics, vol. 88, no. 4, pp. 781-794, 2011.

31. R. Mohammadzadeh, M. Lakestani, Analysis of time-varying delay systems by hybrid of block-pulse functions and biorthogonal multiscaling functions, International Journal of Control, vol. 88, no. 12, pp. 2444-2456, 2015.

32. H.R. Marzban, H. Pirmoradian, A novel approach for the numerical investigation of optimal control problems containing multiple delays, Optimal Control Applications and Methods, vol. 39, no. 1, pp. 302-325, 2018.

33. A. Galindro, D.F. M. Torres, A simple mathematical model for unemployment: a case study in Portugal with optimal control, Statistics, Optimization \& Information Computing, vol. 6, no. 1, pp. 116-129, 2018

34. J. N.C. Gonçalves, H.S. Rodrigues, M.T. T. Monteiro, On the dynamics of a viral marketing model with optimal control using indirect and direct methods, Statistics, Optimization \& Information Computing, vol. 6, no. 4, pp. 633-644, 2018. 